\title{
Molecular simulation of loading-dependent diffusion in nanoporous materials using extended dynamically corrected transition state theory
}

\author{
D. Dubbeldam ${ }^{\text {a) }}$ and E. Beerdsen \\ Van't Hoff Institute for Molecular Sciences, University of Amsterdam, Nieuwe Achtergracht 166, \\ 1018 WV Amsterdam, The Netherlands \\ T. J. H. Vlugt \\ Condensed Matter and Interfaces, Debye Institute, Utrecht University, P.O. Box 80.000, \\ 3508 TA Utrecht, The Netherlands

\section{B. Smit} \\ Van't Hoff Institute for Molecular Sciences, University of Amsterdam, Nieuwe Achtergracht 166, \\ 1018 WV Amsterdam, The Netherlands and Centre Européen de Calcul Atomique et Moleculaire (Cecam), \\ Ecole Normale Supérieure 46, Allée d'Italie, 69007 Lyon, France
}

(Received 1 February 2005; accepted 4 April 2005; published online 16 June 2005)

\begin{abstract}
A dynamically corrected transition state theory method is presented that is capable of computing quantitatively the self-diffusivity of adsorbed molecules in confined systems at nonzero loading. This extention to traditional transition state theory is free of additional assumptions and yields a diffusivity identical to that obtained by conventional molecular-dynamics simulations. While molecular-dynamics calculations are limited to relatively fast diffusing molecules, our approach extends the range of accessible time scales significantly beyond currently available methods. We show results for methane, ethane, and propane in LTL- and LTA-type zeolites over a wide range of temperatures and loadings, and demonstrate the extensibility of the method to mixtures. (C) 2005 American Institute of Physics. [DOI: 10.1063/1.1924548]
\end{abstract}

\section{INTRODUCTION}

Molecular simulation ${ }^{1,2}$ has evolved over the years as a powerful tool to study the equilibrium and transport properties of molecules adsorbed in nanoporous materials. It provides an understanding of the microscopic dynamics underlying the macroscopic properties of industrial interest such as the separation of mixtures of molecules. ${ }^{3}$ The use of zeolites as a means for chemically clean separations can be considered a prime example of how nanomaterials are able to exploit the critical match between the confinement and the shape and size of the adsorbate. One of the difficulties encountered when studying diffusion behavior in zeolites using simulation is that many processes occur outside the time scale accessible to molecular dynamics (MD), which is currently typically limited to diffusion rates in the order of $10^{-12} \mathrm{~m}^{2} / \mathrm{s}$.

New methods have been developed for circumventing this time scale problem. ${ }^{4}$ Systems characterized by a sequence of rare events can be described by transition state theory (TST) methods such as the Bennett-Chandler approach, ${ }^{5,6}$ the method of Ruiz-Montero et al., ${ }^{7}$ path sampling, ${ }^{8}$ transition interface sampling, ${ }^{9,10}$ hyperdynamics, ${ }^{11}$ parallel replica dynamics, ${ }^{12}$ temperatureaccelerated dynamics, ${ }^{13}$ and on-the-fly kinetic Monte Carlo. ${ }^{14}$ In principle, all of these methods have the potential to be orders of magnitude more efficient while still retaining full atomistic detail. In TST approximations one computes a

\footnotetext{
${ }^{a)}$ Author to whom correspondence should be addressed. Electronic mail: d.dubbeldam@uva.nl
}

rate constant between states $A$ and $B$ by computing the equilibrium particle flux through the dividing surface. The dividing surface should uniquely divide two connected states, and in general the TST rate is an upper bound on the exact rate. The exact rate can be recovered by running short MD trajectories from the dividing surface to compute a dynamical correction $(\mathrm{dc}){ }^{1}$

Many groups have worked on the time scale problem for diffusion in confinement. The approach of June et al. ${ }^{15}$ models self-diffusion of xenon and $\mathrm{SF}_{6}$ in silicalite at infinite dilution as a series of uncorrelated jumps between potentialenergy minima (sites). The rate constants for jumping between the sites are converted to diffusivities by generating continuous-time/discrete-space Monte Carlo random walks. The computed diffusivities were reasonably close to the values computed using conventional MD. Snurr et al. ${ }^{16}$ developed a hierarchical approach for predicting isotherms of benzene in silicalite. The method can be applied to other systems when molecules adsorb at well-defined sites. In a subsequent paper, Snurr et al. ${ }^{17}$ investigated the dynamical behavior of benzene using TST. Diffusion paths connecting pairs of potential-energy minima are constructed through saddle points (transition states). Given the rate constants, the selfdiffusivity was computed with a kinetic Monte Carlo simulation. Maginn et al. ${ }^{18}$ presented a hierarchical approach for simulating the diffusion of $n$-alkanes up to $\mathrm{C}_{20}$ in silicalite using modest computational resources. The simulation strategy utilized concepts from Brownian motion theory and transition state theory. Jousse and Auerbach ${ }^{19}$ used TST to compute exact rate coefficients for benzene jumps in $\mathrm{NaY}$ 
zeolite. Forester and Smith ${ }^{20}$ used constrained reaction coordinate dynamics (Bluemoon ensemble) to characterize the free-energy profile of benzene in silicalite- 1 at $300 \mathrm{~K}$ along the mean reaction path for diffusion. The free energies, combined with estimates of the transmission coefficient, were used to obtain rate constants for diffusion between the main adsorption sites. Subsequent kinetic Monte Carlo simulations provided the self-diffusion coefficients. Mossel et $a^{21,22}$ studied the diffusion of benzene and $p$-xylene in zeolite $\mathrm{NaY}$ by means of constrained reaction coordinate dynamics. MD simulations were used to determine the potential of mean force along the coordinate perpendicular to the window connecting two supercages of the zeolite. Diffusion coefficients and activation energies were determined from a hopping model that considers dynamical corrections. Ghorai et al. ${ }^{23}$ estimated the rate of passage of $\mathrm{CCl}_{4}$ through the eight-ring window in a model of zeolite A by combining a direct evaluation of the free-energy profile and an adaptation of the rare events method. The system contains on average one particle per cage, and because particle-particle interactions rarely occur under this condition the free energy is evaluated from the one-particle partition function. The self-diffusion of ethane in cation-free (LTA)-type zeolite has been studied by Schüring et $a l .{ }^{24}$ using MD and TST (without dynamical correction) for various temperatures. The bare TST jump rates were similar to the MD jump rates (where also the MD results were not corrected for short-time recrossings). Dubbeldam and co-workers ${ }^{25,26}$ applied dynamically corrected transition state theory (dcTST) to study abnormal diffusion of linear alkane molecules $\left(\mathrm{C}_{1}-\mathrm{C}_{20}\right)$ in ERI-, CHA-, and LTAtype zeolites at infinite dilution. The exceptionally slowdiffusion rates required the combination of rare-event TST techniques with the configurational-bias Monte Carlo (CBMC) algorithm. ${ }^{1,27}$ The diffusivities were evaluated on a lattice spanned by the cage centers.

It is important to note that these works have been performed at infinite dilution, even though many of the processes of industrial importance occur at nonzero loading. ${ }^{28}$ Only a limited number of studies deal with nonzero loading. Tunca and Ford ${ }^{29}$ used multidimensional TST to obtain the hopping rate of adsorbates from an $\alpha$-cage in LTA-type zeolite as a function of loading. Various approximations were applied to make the simulations computationally feasible. In a subsequent study ${ }^{30}$ the limitations of an empty receiving cage and the use of the Widom insertion method were avoided. Recently, Tunca and Ford presented a new hierarchical approach to the molecular modeling of diffusion and adsorption at nonzero loading in microporous materials. ${ }^{31}$ Although adsorption was well represented, the coarsegrained self-diffusivity data under predicted the diffusivity at low loading, while significantly over predicting the diffusivities at higher loadings, in comparison to conventional MD.

Coarse-grained kinetic Monte Carlo (kMC) studies have pointed at the difficulties of computing an elementary hopping rate, taking into account the various correlations induced by particle-particle interactions. ${ }^{31,32}$ Very recently, Beerdsen et al..$^{33}$ extended the dcTST Bennett-Chandler approach to include diffusion of molecules at nonzero loading.
It was shown that the particle-particle correlations can be taken into account by a proper definition of an effective hopping rate of a single particle. The self-diffusivity was computed directly by computing the hopping rate of a molecule over a typical length scale given by the smallest repeating zeolite structure, i.e., from the center of cage $A$ to the center of cage $B$. The use of kinetic Monte Carlo and its underlying assumptions are therefore avoided. Implicitly one integrates over the whole volume of a cage and hence all adsorption sites in the cage, irrespective whether these are well defined or not. All other particles are regarded as a contribution to the external field exerted on this tagged particle. The dcTST extension to finite loadings yielded excellent agreement with that obtained by conventional MD simulations and is applicable in any system containing high free-energy barriers and for any type of guest molecule. In this work, we elaborate on the concepts introduced by Beerdsen et al., and show results for methane, ethane, and propane in LTL- and LTA-type zeolites over a wide range of temperatures and loadings.

The remainder of the paper is organized as follows. In Sec. II we explain the used methods and concepts. First, the force field is described, and we present shortly the canonical MD algorithm focusing on maintaining temperature control and obtaining diffusion coefficients. Next, we discuss some concepts from random walk theory, e.g., jump rates and memory effects. We show that TST is fully compatible and consistent with random-walk theory and present our dcTST at nonzero loading. Section III starts with the results on the infinite dilution case. Two different sets of parameters from literature are used and the difference indicates that the physics of adsorption and diffusion in zeolites is often highly parameter dependent. The main emphasis of the paper lies on the diffusivity results of methane, ethane, and propane in LTL- and LTA-type zeolites using dcTST compared to MD as a function of loading. For LTA-type zeolite we present additional results of a mixture of methane and ethane. We end with a general discussion on lattices, correlations, and dcTST in Sec. IV, and conclusions in Sec. V.

\section{METHODOLOGY}

\section{A. Force field parameters for adsorption and diffusion of alkanes in siliceous nanoporous materials}

Zeolites are confined systems with pore sizes comparable to the molecular size. Adsorption in cation-free zeolite structures usually takes place at specific sites with little or no electric field. For this reason the united-atom model ${ }^{34}$ seems the most straightforward choice. We consider the $\mathrm{CH}_{x}$ groups as single, chargeless interaction centers with their own effective potentials. The beads in the chain are connected by harmonic bonding potentials. A harmonic cosine bending potential models the bond bending between three neighboring beads. The Lennard-Jones potentials are shifted and cut at $12 \AA$. Analytical tail corrections are not applicable in zeolites. ${ }^{35}$ A truncated and shifted potential is equally suitable to Monte Carlo and molecular dynamics. Flexibility of the framework is not an issue for adsorption of linear and branched alkanes. ${ }^{36}$ For methane in LTA-type zeolite it was 
TABLE I. Force field of Dubbeldam et al. (Refs. 39 and 40) for guest-host and guest-guest interactions of hydrocarbons in cation-free nanoporous materials. Lennard-Jones parameters, $\epsilon / k_{B}[\mathrm{~K}]$ in the top of each field, $\sigma[\AA]$ in the bottom of each field, bond and bend parameters.

\begin{tabular}{rlllll}
\hline \hline & $\mathrm{O}$ & $\mathrm{Si}$ & $\mathrm{CH}_{4}$ & $\mathrm{CH}_{3}$ & $\mathrm{CH}_{2}$ \\
\hline $\mathrm{CH}_{4}$ & 115.00 & $\ldots$ & 158.50 & 130.84 & 94.21 \\
& 3.47 & & 3.72 & 3.74 & 3.84 \\
$\mathrm{CH}_{3}$ & 93.00 & $\ldots$ & 130.84 & 108.00 & 77.7 \\
& 3.48 & & 3.74 & 3.76 & 3.86 \\
$\mathrm{CH}_{2}$ & 60.50 & $\ldots$ & 94.21 & 77.77 & 56.00 \\
\multirow{3}{*}{ Bond } & 3.58 & & 3.84 & 3.86 & 3.96 \\
& $U^{\text {bond }}=\frac{1}{2} k_{1}\left(r-r_{0}\right)^{2}$ & & & \\
\multirow{2}{*}{ Bend } & $k_{1} / k_{B}=96500 \mathrm{~K} / \AA^{2}, r_{0}=1.54 \AA$ & & & \\
& $U^{\text {bend }}=\frac{1}{2} k_{2}\left(\cos \theta-\cos \theta_{0}\right)^{2}$ & & & \\
& $k_{2} / k_{B}=62500 \mathrm{~K}, \theta_{0}=114^{\circ}$ & & &
\end{tabular}

found that self-diffusion coefficients obtained with flexible and with rigid lattices are also practically the $\operatorname{same}^{37}$ (in the Discussion section we will further comment on this). The interactions between the rigid framework and the guest molecules are assumed to be dominated by the oxygen atoms. ${ }^{38}$ The interaction parameters of alkanes listed in Table I for use in molecular simulations of confined systems are obtained uniquely and accurately through fitting on experimental isotherms with inflection points. ${ }^{39,40}$ Recently, it was shown that these parameters also give near-quantitative agreement for collective diffusivities for ethane in silicalite compared to neutron-scattering experiments. ${ }^{41}$ Details on the simulations can be found in Refs. 39 and 42. The parameters listed in Table II are used mainly for comparison with the simulations of Schüring et al. ${ }^{24,43}$ and because their very small size parameters enhance diffusion by two orders of magnitude for ethane in LTA-type zeolite compared to the parameters of Dubbeldam et al. Although the parameters used by Schüring et al. are probably less realistic in our opinion, they are convenient to compare the diffusion of ethane from a simulation-method point of view by MD and dcTST in LTAtype zeolite.

\section{B. Molecular dynamics (MD)}

In MD simulations, ${ }^{1,2,44}$ successive configurations of the system are generated by integrating Newton's laws of motion, which then yields a trajectory that describes the positions, velocities, and accelerations of the particles as they vary with time. The self-diffusion coefficients $D_{S}^{\alpha}$ in the direction $\alpha=x, y, z$ are computed by taking the slope of the mean-squared displacement (msd) at long times

TABLE II. Force field LJ parameters used by Schüring et al. for ethane in cation-free LTA-type zeolite (Ref. 24). Lennard-Jones parameters, $\epsilon / k_{B}[\mathrm{~K}]$ in the top of each field, $\sigma[\AA]$ in the bottom of each field, bond and bend parameters.

\begin{tabular}{cccc}
\hline \hline & $\mathrm{O}$ & $\mathrm{Si}$ & $\mathrm{CH}_{3}$ \\
\hline \multirow{2}{*}{$\mathrm{CH}_{3}$} & 142 & 82 & 104 \\
& 3.17 & 2.12 & 3.78 \\
\multirow{3}{*}{ Bond } & $U^{\text {bond }}=\frac{1}{2} k_{1}\left(r-r_{0}\right)^{2}$ & & \\
& $k_{1} / k_{B}=96500 \mathrm{~K} / \AA^{2}, r_{0}=1.54 \AA$ & \\
\hline \hline
\end{tabular}

$$
D_{S}^{\alpha}=\frac{1}{2 N} \lim _{t \rightarrow \infty} \frac{d}{d t}\left\langle\sum_{i=1}^{N}\left[r_{i \alpha}(t)-r_{i \alpha}(0)\right]^{2}\right\rangle,
$$

where $N$ is the number of molecules, $t$ the time, and $r_{i \alpha}$ the $\alpha$ component of the center of mass of molecule $i$. Equivalently, $D_{\alpha}$ is given by the time integral of the velocity autocorrelation function

$$
D_{S}^{\alpha}=\frac{1}{N} \int_{0}^{\infty}\left\langle\sum_{i=1}^{N} v_{i \alpha}(t) v_{i \alpha}(0)\right\rangle d t,
$$

where $v_{i \alpha}$ is the $\alpha$ component of the center-of-mass velocity of molecule $i$. A separation of time scales occurs for interacting particles roughly at the times between particle-particle and particle-zeolite collisions (Fig. 1). The mean-squared displacement thus bends over to attain a different slope, and we are interested in the long-time diffusion coefficient. The collective diffusion coefficients $D_{C}^{\alpha}$ are given by

$$
D_{C}^{\alpha}=\frac{1}{2 N} \lim _{t \rightarrow \infty} \frac{d}{d t}\left\langle\left\{\sum_{i=1}^{N}\left[r_{i \alpha}(t)-r_{i \alpha}(0)\right]\right\}^{2}\right\rangle
$$

and

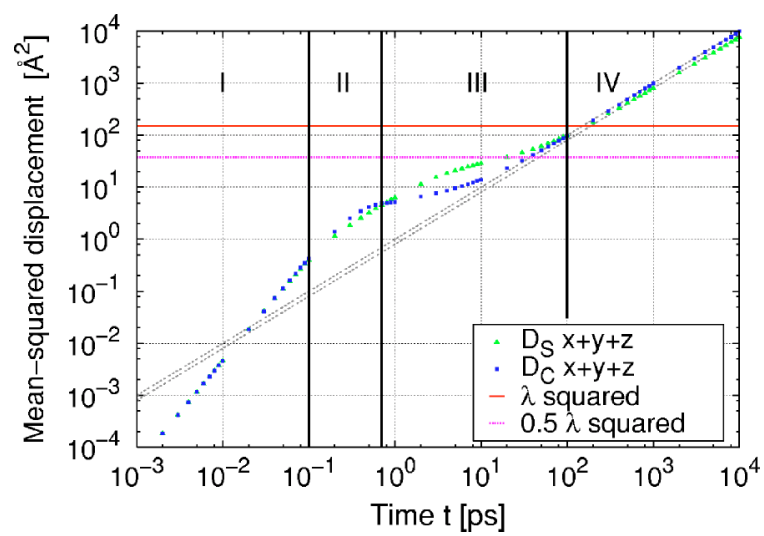

FIG. 1. Mean-squared displacement of self-diffusivity $D_{S}$ and collective diffusivity $D_{C}$ for methane in LTA-type zeolite at $300 \mathrm{~K}$ at an average loading of 8 molecules/cage. We can identify four distinct regimes (see text). For reference, we show $\left(\frac{1}{2} \lambda\right)^{2}$ and $\lambda^{2}$, with $\lambda=12.2775 \AA$ the cage-center to cage-center lattice distance. The dotted lines are of slope unity and indicate normal diffusive behavior. 


$$
D_{C}^{\alpha}=\frac{1}{N} \int_{0}^{\infty}\left\langle\left[\sum_{i=1}^{N} v_{i \alpha}(t)\right]\left[\sum_{i=1}^{N} v_{i \alpha}(0)\right]\right\rangle d t
$$

There are several forms of collective diffusion, the definition given here is in zeolite-literature often referred to as corrected diffusivity. The inclusion of a thermodynamic factor results in the so-called transport diffusivity, which is directly related to the macroscopic Fickian diffusion. The removal of the thermodynamic effect results in a slightly less loading dependent behavior. Collective diffusivity measures the transport of mass and the decay of density fluctuations in the system, while self-diffusion measures the diffusive motion of a single particle. The directionally averaged diffusion coefficient is given by

$$
D=\frac{D_{x}+D_{y}+D_{z}}{3} .
$$

Note that in simple fluids there is only a time scale separation for the self-motion, not for the collective motion. In nanoporous materials, both the displacements of the single particles as well as the displacements of the total center of mass are restricted by the confinement and the time scale separation is also present in collective diffusion. This is very much related to the diffusion of polymers in melts where similar time scale separations occur. ${ }^{45}$ In Fig. 1 we show the mean-squared displacements of the self- and collective motions at $300 \mathrm{~K}$ of methane in LTA-type zeolite at an average loading of 8 molecules/cage. Several regimes can be identified for this system,

At very short time scales both the self- and collective motions are ballistic, and the msd is proportional to $t^{2}$. While initially the same, the msd of self-motion is lowered in comparison to the collective motion due to the backcorrelation mechanisms that also occur in simple fluids. The onset of regime II is signaling the average mean free time before particles collide.

(III) Regime three is dominated by a confinement effect and particles have not yet been able, on average, to hop to the next confinement. The msd of single particles is restricted to approximately the cage size squared, but results in cancellation for the collective behavior. The msd of self-motion is therefore higher than for collective motion.

(IV) With increasing times the particles are increasingly able to leave the confinement, and both self- and collective motions increase eventually to a linear diffusive regime IV. Here, particles originating from different cages start to collide, and self-motion is again lowered in comparison with collective motion. For collective motion the onset of this hydrodynamical regime is a combined effect of confinement and the time particles start to leave the cage (because here, a change in the collective motion can only be accomplished by cage-to-cage hops of single particles). The onset for self-motion for cage/window-type systems is the cage-size squared, i.e., the average time for a particle to leave a cage.
The calculation of the diffusion coefficients requires much memory and CPU power, especially when fluctuations decay slowly. The order- $n$ algorithm to measure correlations allows us to measure fast and slow dynamics simultaneously at a minimal computational cost by using adjustable sampling frequencies. ${ }^{1}$ The order- $n$ scheme is equally accurate as the conventional scheme but the saving in memory as well as CPU time is significant for computing the mean-squared displacements at long times.

In a conventional $N V E$ molecular-dynamics simulation, the total energy $E$, the number of particles $N$, and the volume $V$ are constant. Hence, MD measures the (time) averages in the microcanonical ensemble, while in a conventional Monte Carlo simulation the canonical ensemble ( $N V T$ ensemble) is probed. The extended Lagrangian approach has become one of the most important tricks for MD in the NVT ensemble and is completely dynamic in origin. The Nosé-Hoover Chain (NHC) formulation extends the Lagrangian with additional coordinates and velocities containing the system to a constant temperature $N V T$ ensemble. We use the NHC method as implemented by Martyna et al. ${ }^{46}$ in which the dynamics is still reversible. The instantaneous kinetic temperature fluctuates, but the probability to find the system in a given energy state follows the Maxwell-Boltzmann distribution.

For the instantaneous temperature we measure the total kinetic energy of the system and divide this by the number of degrees of freedom $N_{f}(=3 N-3$ for a system of $N$ particles with fixed total momentum)

$$
k_{B} T(t)=\sum_{i=1}^{N} \frac{m_{i} v_{i}^{2}(t)}{N_{f}} .
$$

The disadvantage of most methods for working at constant temperature is that the dynamics is changed in an artificial way. Because in our simulations we do not have photons or electrons, i.e., the system is mechanical, heat is transported at the speed of sound or slower. However, most thermostat methods have a coupling constant, e.g., the masses of the NHC and the effect of the thermostat on the particles is instantaneous. The NHC masses should therefore be chosen as large as possible to alter the dynamics as little as possible. If this is taken care of, the nonphysical effects for dynamical properties will be of order $(1 / N)$ in general.

Figure 2 shows the importance of adequate temperature control. Ideally, a flexible zeolite would provide excellent thermostatting of adsorbed molecules (see Fig. 18 in the Discussion, Sec. IV). However, for computational reasons many authors keep the framework rigid, and the thermostatting issue arises. In the $N V E$ ensemble the particles do not exchange energy with the heat bath and thermalization occurs through mutual interactions between the adsorbates. Moreover, rather unphysical ballistic motion may occur, and particles may be stuck in local free-energy barriers. The nonphysical effects of the NHC thermostat using a single molecule are clearly present in both MD and dcTST. We note that at high temperatures the thermostat effects are small, but they become significant at very low temperatures. This implies that single-particle diffusion coefficients or correlations 


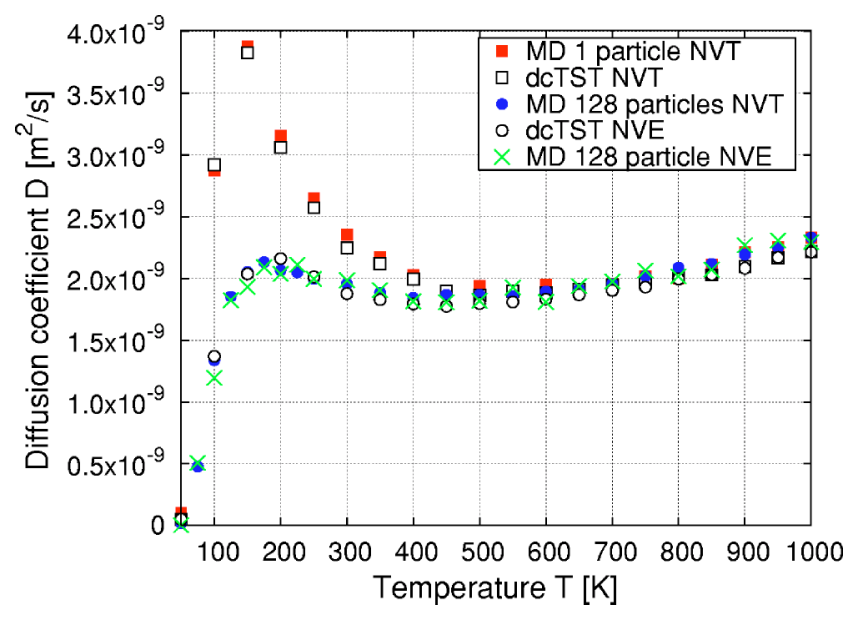

FIG. 2. Self-diffusion of ethane at infinite dilution in LTA-type silica using the parameter sets of Schüring et al. The infinite dilution case using MD is simulated by switching the ethane intermolecular forces off, i.e., the particles do not interact (except through the thermostat in NHC-NVT simulations).

should be computed in the NVE ensemble, unless a sufficient number of particles is used. At infinite dilution this can be accomplished by switching the intermolecular forces off, i.e., the particles do not interact except through the thermostat in NVT NHC simulations. However, even if this is taken care of, the NHC method is only capable of maintaining adequate temperature control in equilibrium, and therefore breaks down in the limit of high potential-energy barriers. ${ }^{47}$ Entropical barriers (e.g., due to constrictions and apertures in zeolite cages and channels) represent no problem.

To prepare the system at the desired temperature in an equilibrium configuration we initialize the system by the following procedure:

- $N$ molecules are inserted into the framework at random positions as long as no overlaps occur with the framework or other particles, and as long as the positions are accessible from the main cages and channels.

- During the initializing period we perform an NVT MC simulation to rapidly achieve an equilibrium molecular arrangement.

- After the initialization step, we assign all the atoms velocities from the Maxwell-Boltzmann distribution at the desired average temperature. The total momentum of the system is set to zero. Next, we equilibrate the system further by performing an NVT MD simulation using the NHC thermostat.

- The equilibration is completed and during the production run we collect statistics using either the $N V E$ or $N V T$ ensemble. Following this equilibration procedure, the average temperature using $N V E$ over the entire production period is usually within a few Kelvin of the desired average temperature, while $N V T$ would give the exact desired average temperature if simulated sufficiently long.

\section{Lattice random-walk theory}

Diffusive motion of particles occurs by a series of discrete steps separated by elastic collisions, localized vibrations, and short shuffles. Diffusion is an irreversible macroscopic process, but is actually comprised of reversible microscopic steps, and may be well described by randomwalk theory. A random walk is a simple mathematical model for the movement of a particle on a lattice under the influence of some random or stochastic force affecting its direction of motion. It is particularly attractive because in many instances analytical solutions can be worked out for both static as well as dynamic properties. From the internal (crystal) structure a lattice can be constructed that determines the lattice topology and the lattice distances. The dynamics of the random walk is uniquely determined once the jumping frequencies $k_{i}$ for a lattice direction $i$ are specified. A jump frequency is defined as

$$
k=\frac{\langle\text { number of successful hops }\rangle}{\text { unit of time }} .
$$

The total jumping frequency $k_{\text {tot }}$ is related to the specific jumping frequencies $k_{i}$ for a given structure by a summation over the lattice connectivity $Z$ :

$$
k_{\mathrm{tot}}=\sum_{i=1}^{Z} k_{i} \text {. }
$$

For a truly random jump each of the possible jump directions is chosen with equal probability. The probability that the new lattice site is empty does not enter into any equation (the particles can overlap). The expected value $\langle\mathbf{r}(t)\rangle=0$, and the chemical potential driving force $\nabla \mu=0$ for a simple regular random walk (necessary for the measurement of the selfdiffusivity $D_{S}$ ). However, in real systems, jumps are usually correlated by defined interactions between jumping particles.

Let $k_{i}$ be the average frequency that a random walker (an atom or molecule) jumps for lattice vector $\boldsymbol{\lambda}_{i}$, and $\mathbf{r}(t)$ is the position of a particular random walker. The position of a particle (relative to the starting position) after a time $t$ (or $n=k t$ hops) will be

$$
\mathbf{r}(t)=\sum_{i=1}^{n} \boldsymbol{\lambda}_{i} .
$$

In primitive cubic crystals there exists one lattice site per unit cell surrounded by $Z=6$ neighbors, the lattice vectors are $\boldsymbol{\lambda}_{i}=\lambda \hat{\mathbf{e}}_{i}$ with

$\hat{\mathbf{e}}_{i}=(\{1,0,0\},\{0,1,0\},\{0,0,1\},\{-1,0,0\},\{0,-1,0\},\{0,0,-1\})$.

The distance between two particles should increase with time, which is measured by the spread of the distribution $\langle\mathbf{r}(t)\rangle$,

$$
\left\langle\mathbf{r}^{2}(t)\right\rangle=\left\langle\sum_{i=1}^{n} \boldsymbol{\lambda}_{i} \cdot \boldsymbol{\lambda}_{i}+2 \sum_{i=1}^{n-1} \sum_{j=i+1}^{n} \boldsymbol{\lambda}_{i} \cdot \boldsymbol{\lambda}_{j}\right\rangle,
$$

written as a sum of diagonal and off-diagonal terms. 
For the one-dimensional lattice, the two-dimensional square lattice, and the three-dimensional cubic lattice, all the jumping frequencies $k_{i}$ and jump vectors are equivalent. Using the relationship

$$
\boldsymbol{\lambda}_{i} \cdot \boldsymbol{\lambda}_{j}=\left|\boldsymbol{\lambda}_{i}\right|\left|\boldsymbol{\lambda}_{j}\right| \cos \Delta \phi_{i j}=\lambda^{2} \cos \Delta \phi_{i j},
$$

where $\Delta \phi_{i j}=-\Delta \phi_{j i}$ is the angle between the $i$ th and $j$ th jump vectors, we find

$$
\left\langle\mathbf{r}^{2}(t)\right\rangle=n \lambda^{2}\left(1+\frac{1}{n}\left\langle\sum_{i=1}^{n} \sum_{j=1}^{n} \cos \Delta \phi_{i j}\right\rangle\right),
$$

and we can write for $n \rightarrow \infty$

$$
\left\langle\mathbf{r}(t)^{2}\right\rangle=n f \lambda^{2}=Z k_{i} f \lambda^{2} t,
$$

where $f$ denotes the correlation factor

$$
f \equiv 1+\frac{1}{n}\left\langle\sum_{i=1}^{n} \sum_{j=1}^{n} \cos \Delta \phi_{i j}\right\rangle .
$$

Applying Einstein's equation

$$
\left\langle\mathbf{r}(t)^{2}\right\rangle=2 d D t
$$

yields

$$
\begin{aligned}
& D=\frac{Z}{2 d} f k_{i} \lambda^{2}=f k_{A \rightarrow B} \lambda^{2}, \\
& =\frac{1}{2 d} f k_{\mathrm{tot}} \lambda^{2} .
\end{aligned}
$$

This relates the macroscopic self-diffusivity to the jump frequency $k$, lattice hop distance $\lambda$, and correlation factor $f$. The basic assumption of the random-walk model is the quick loss of memory of the molecules between consecutive jumps, i.e., a molecule will proceed with a probability independent of its history. The correlation factor $f$ contains all memory effects, arising from ordering and interparticle interactions.

The regular random walk has no "memory" of the previous step when determining the current one. This feature can be applied to a wide range of physical problems, but there are a number of other interesting problems for which this is not the case. In a persistent walk, the transition (or step) probability depends upon the previous transition, and a particle has a retention to the directional over a certain number of trajectory steps. In order for a simplistic regular lattice model to be valid, the loss of memory is an important condition that has to be satisfied.

\section{Correlations}

The collective diffusivity contains all the dynamical correlations. Here, the motion results from the jumps of different particles at different times. In contrast, for self-diffusivity the motion results from the jumps of a tagged particle at different times. Memory effects ${ }^{48,49}$ have a tendency to decrease $D_{S}$ with respect to $D_{C}$, indicating it is somehow related to the well-known backcorrelation mechanism where a diffusing particle has a higher probability to jump backwards than in any other direction, simply because the originating site is guaranteed to be empty. Most of the memory effect arise

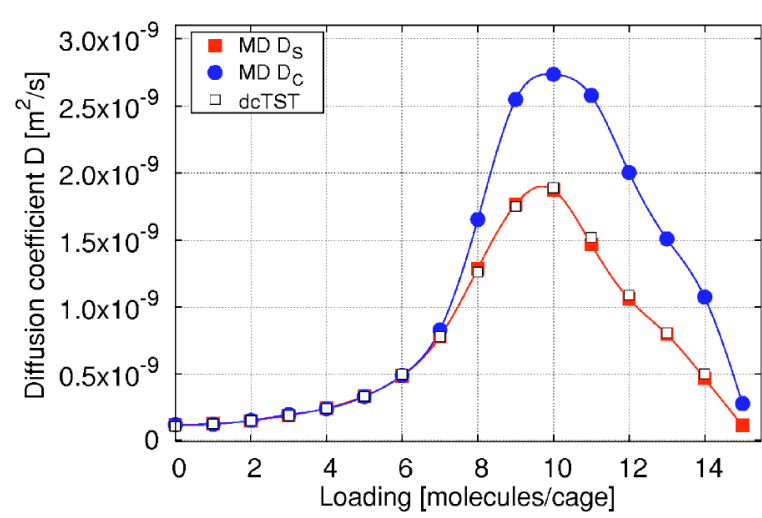

FIG. 3. Self-diffusivity $D_{S}$ and collective diffusivity $D_{C}$ for methane in LTA-type zeolite at $300 \mathrm{~K}$ as a function of loading.

from ordering and interparticle interactions, the latter giving the leading contribution. Figure 3 shows the self- and collective diffusivities of methane in LTA-type zeolite at $300 \mathrm{~K}$ as a function of loading. In the low loading limit both diffusivities converge, because particle-particle interactions vanish. However, at a loading of 7 molecule/cage or higher the correlations are clearly visible. Although in principle conventional MD captures all relevant correlations, the dcTST method by Beerdsen et al. not only captures these correlations correctly but is also suitable for systems with large free-energy barriers. ${ }^{33}$ These correlations, originating from particle-particle interactions, are significant at higher loadings as is evidenced by the large difference between collective and self-diffusivities.

Memory effects are stronger on single-particle motion than on the collective motion, where most of the backcorrelations cancel out. For a Langmuir gas, where the only interaction is the site exclusion, they cancel out exactly. Correlations between successive jumps can be studied by considering directional correlations between two jumps separated by $m$ previous jumps by a tagged particle. For a Langmuir gas the factor $f$ reduces in the high loading limit to $^{48}$

$$
f=\frac{1+\langle\cos \phi\rangle}{1-\langle\cos \phi\rangle},
$$

as a correlation factor for vacancy diffusion, where $\phi$ is the average angle between two consecutive single-particle jumps. This equation assumes that the predominant memory contribution comes from the backcorrelation between two consecutive single-particle jumps $(m=1)$. Equation (19) is very much related to the end-to-end distance of an isolated, infinitely long, hypothetical model chain comprised of bonds of fixed lengths joined with fixed bend angles. ${ }^{50}$ Unlike the freely joined case, the fixing of the angles $\phi$ imposes correlations.

A particle residing in a lattice point once in a while jumps to a neighboring site. If thermalization occurs, we call it a single jump, otherwise we speak of a long jump or a multijump. These kinetic correlations become important at low loadings and in channel-type structures with smooth walls, e.g., carbon nanotubes. However, for entropydominated barriers (e.g., methane and ethane in LTA-type 
zeolites) one can usually neglect kinetic correlations.

\section{E. Dynamically corrected transition state theory (dcTST) at infinite dilution}

At infinite dilution and sufficient dissipation the correlation factor $f=1$ (there are no memory effects), and Eq. (17) reduces to

$$
D=k_{i} \lambda^{2}=k_{A B} \lambda^{2} .
$$

The lattice distance $\lambda$ is fixed and a property that can be obtained from crystal X-ray scattering experiments. Therefore, Eq. (20) defines $k_{i}$ as the hopping rate from lattice point $A$ (in equilibrium) to a neighboring lattice point $B$ (in equilibrium). Note that an attempt of a hop is always successful, and that a particle cannot have a position in between the lattice points, i.e., the jump is instantaneous and discrete. In principle, one could use MD simulations to determine this rate by computing the average residence time that a particle is in a cage. However, such a computation using MD proves cumbersome. Firstly, an $A$-to- $B$ order parameter has to be defined, secondly, a criteria should be used to distinguish unsuccessful hops on a very short time scale from the successful $A B$ hop on a much longer time scale (the equilibrium one), and thirdly, very few trajectories will involve motion from exactly point $A$ to point $B$.

Dynamically corrected transition state theory overcomes these problems by computing precisely what we need: the hopping rate from lattice point $A$ (in equilibrium) to a neighboring lattice point $B$ (in equilibrium). In other words, dcTST is fully compatible and complementary to lattice random-walk theory. We consider a system which can be in two stable states, $A$ and $B$. The reaction coordinate, a parameter that indicates the progress of the diffusion event from region $A$ to region $B$, is denoted by $q$. Here, $q$ is a function of the Cartesian coordinates, $\dot{q}$ denotes its time derivative, $q^{*}$ is the location of the dividing surface, and $q_{A}, q_{B}$ are the minima of the free energy corresponding to states $A$ and $B$, respectively. In general, the reaction coordinate $q$ is a function of the configuration of the whole system, i.e., $q$ $=q\left(\mathbf{r}_{1}, \ldots, \mathbf{r}_{N}\right)$. However, we can choose $q$ as the position of one of the atoms of the diffusing molecules. ${ }^{25}$ We introduce two characteristic functions $n_{A}$ and $n_{B}$ that measure whether the system is in state $A$ or $B$. A possible and often used definition is

$$
\begin{aligned}
& n_{A}=\theta\left(q^{*}-q\right), \\
& n_{B}=\theta\left(q-q^{*}\right),
\end{aligned}
$$

where $\theta$ is the Heaviside function $\theta(x)$, which has a value zero for $x<0$ and a value of unity for $x \geqslant 0$. With these definitions the transition rate $k_{A \rightarrow B}$ is given by ${ }^{7}$

$$
k_{A \rightarrow B}=\underbrace{\frac{\left\langle\delta\left(q^{*}-q\right)\right\rangle}{\left\langle\theta\left(q^{*}-q\right)\right\rangle}}_{P_{\in A}\left(q^{*}\right)} \underbrace{\frac{\left\langle\dot{q}(0) \delta\left[q^{*}-q(0)\right] \theta\left[q(t)-q^{*}\right]\right\rangle}{\left\langle\delta\left[q^{*}-q(0)\right]\right\rangle}}_{R(t)},
$$

where $\delta$ is the Dirac delta function, $P_{\in A}\left(q^{*}\right)$ is the equilibrium probability density of finding the system at the top of the barrier divided by the equilibrium probability of finding it at state $A$, and where $R(t)$ is the averaged particle flux at the top of the barrier multiplied by the probability that the system ends up in state $B$ at time $t$. From detailed balance follows

$$
\frac{k_{A \rightarrow B}}{k_{B \rightarrow A}}=\frac{\left\langle n_{B}\right\rangle}{\left\langle n_{A}\right\rangle},
$$

where $\left\langle n_{A}\right\rangle$ is the equilibrium mole fraction of particles in state $A$,

$$
\begin{aligned}
& \left\langle n_{A}\right\rangle=\frac{\int_{A} e^{-\beta F(q)} d q}{\int_{A+B} e^{-\beta F(q)} d q}, \\
& \left\langle n_{B}\right\rangle=\left\langle 1-n_{A}\right\rangle .
\end{aligned}
$$

The expression Eq. (23) is rigorously correct for arbitrary crossings, provided that (a) the actual crossing time is negligible compared to the time a particle spends inside the cage, i.e., there is a large separation in time scales. This condition is satisfied when the free-energy barrier is much larger than $k_{B} T$; (b) the velocity distribution at the dividing surface is known. (The order parameter $q$ is taken to be the position of a particle and therefore $\dot{q}$ is simply the velocity of a particle. In TST is it assumed that the particles on top of the barrier are in equilibrium and hence these velocities follow directly from the Maxwell-Boltzmann distribution.)

At infinite dilution, the molecules perform a random walk on a lattice spanned by the cage centers. The transmission rates are easily converted to diffusion coefficients if the jump distance and the number of equivalent diffusion paths are known.

It turns out that Eq. (23) can be written as a product of a static and a dynamic term.

- The probability $P_{\in A}\left(q^{*}\right)$ of finding the system at the top of the barrier is a time-independent equilibrium quantity and can be computed explicitly,

$$
P_{\in A}\left(q^{*}\right)=\frac{\left\langle\delta\left(q^{*}-q\right)\right\rangle}{\left\langle\theta\left(q^{*}-q\right)\right\rangle}=\frac{e^{-\beta F\left(q^{*}\right)}}{\int_{\text {cage } \mathrm{A}} e^{-\beta F(q)} \mathrm{d} q},
$$

where $F(q)$ is the free energy as a function of the diffusion path $q$.

- The flux $R(t)$ through the dividing surface is a conditional average, namely, the product $\dot{q}(0) \theta\left[q(t)-q^{*}\right]$, given that $q(0)=q^{*}$. Using the assumption that the velocities of the atoms follow the Maxwell-Boltzmann distribution, we can estimate from kinetic theory the long-time value of $R(t)$ by $\left\langle\frac{1}{2}|\dot{q}|\right\rangle=\sqrt{k_{B} T / 2 \pi m}$, where $m$ is the mass of the segments of the particle involved in the reaction coordinate (the total mass of the particle if the center of mass is used or the mass of only one segment if the reaction coordinate is a single segment such as the middle bead in a molecule). Transition state theory predicts a crossing rate $k_{A \rightarrow B}^{\mathrm{TST}}$ given by 


$$
k_{A \rightarrow B}^{\mathrm{TST}}=\sqrt{\frac{k_{B} T}{2 \pi m}} \frac{e^{-\beta F\left(q^{*}\right)}}{\int_{\text {cage A }} e^{-\beta F(q)} \mathrm{d} q} .
$$

Calculating TST rate constants is therefore equivalent to calculating free-energy differences.

The TST particle flux estimation $\sqrt{k_{B} T / 2 \pi m}$ contains spurious crossings, i.e., some particles that cross the transition state from $A$ in reality would fail to equilibrate in $B$. The correction $\kappa(t)$ is defined as the ratio between the real rate and the TST expression,

$$
\kappa(t) \equiv \frac{k_{A \rightarrow B}(t)}{k_{A \rightarrow B}^{\mathrm{TST}}}=\frac{\left\langle\dot{q}(0) \delta\left[q(0)-q^{*}\right] \theta\left[q(t)-q^{*}\right]\right\rangle}{\left\langle\frac{1}{2}|\dot{q}(0)|\right\rangle} .
$$

It is the probability that a particle that starts with an initial velocity $\dot{q}$ from the dividing surface will, in fact, cross the barrier, and therefore $\kappa(t)$ corrects for trajectories which cross the transition state from $A$ but fail to equilibrate in $B$. The numerator in Eq. (29) counts trajectories with a positive, but also with a negative weight. It can be shown that $\lim _{t \rightarrow 0^{+}} \kappa(t)=1$ and $\lim _{t \rightarrow 0^{+}} k_{A \rightarrow B}(t)=k_{A \rightarrow B}^{\mathrm{TST}}$. There is a large separation of time scales. The transmissions are completed in a time much less than the time to react, and Eq. (29) will reach a plateau value $\kappa$. For classical systems $0<\kappa \leqslant 1$ and Eq. (28) is corrected as

$$
k_{A \rightarrow B}=\kappa k_{A \rightarrow B}^{\mathrm{TST}} .
$$

Standard molecular dynamics (MD) yields the transmission coefficients, a separate MC simulation is used to generate the starting configurations. The reaction coordinate is restricted to the dividing surface $q^{*}$. The MC moves involved are translations of the reaction bead in the plane of the dividing surface and complete regrowing of the molecule starting from the restricted bead. Subsequently, the transmission coefficient is calculated by standard MD in the NVE ensemble. The beads are given independent velocities, corresponding on average to the desired temperature, by sampling from the Maxwell-Boltzmann distribution.

In the Bennett-Chandler approach it is sufficient to assign the barrier position $q^{*}$ inside the barrier region. The result of the scheme does not depend on the specific location, although the statistical accuracy does. If the dividing surface is not at the top of the barrier the probability of finding a particle will be higher than at the optimal $q^{*}$, but the fraction of the particles that actually cross the barrier will be less than predicted by transition state theory.

\section{F. Importance-sampled MD at infinite dilution}

The approach of $\kappa(t)$ to its plateau value can be quite slow. ${ }^{7}$ Moreover, in the case of diffusive barrier crossings the transmission coefficient is quite small and as a consequence many trajectories have to be generated for an accurate value of $\kappa$. The Bennett-Chandler approach becomes inefficient for systems with low transmission coefficients because the scheme employs the noisy $\theta$ function to detect what state the system in. ${ }^{51}$ The scheme can be improved by constructing a more continuous detection function. More importantly, using the free-energy we can compensate approximately for the effect of the free-energy barrier. This leads to a more or less uniform tagged-particle density distribution over the entire range of $q$. However, only trajectories starting in the barrier region yield relevant information and therefore a weighting function $w(q)$ is applied, restricting the sampling to the barrier region.

A general expression from transition state theory for the rate of hopping from region $A$ to region $B$ over a barrier is: ${ }^{7}$

$$
k_{A \rightarrow B}=\frac{1}{\left\langle n_{A}\right\rangle_{\mathrm{eq}}}\left\langle\dot{q}(0) n_{B}(t) \frac{\partial \chi[q(0)]}{\partial q}\right\rangle,
$$

where $\chi(q)$ is a dimensionless function describing the initial distribution function

$$
\rho(q, t=0)=\rho_{\mathrm{eq}}(q) \chi(q) .
$$

The initial distribution $\chi(q)$ can be approximated well by the steady-state distribution determined from the Fokker-Planck equation

$$
\chi(q)=\frac{1}{\left\langle n_{A}\right\rangle}\left[1-\frac{\int_{q_{A}}^{q} e^{\beta F\left(q^{\prime}\right)} d q^{\prime}}{\int_{q_{A}}^{q_{B}} e^{\beta F\left(q^{\prime}\right)} d q^{\prime}}\right],
$$

and varies rapidly with $q$ in the barrier region and slowly elsewhere, so that $\partial \chi(q) / \partial q$ selects initial configurations in the barrier region

$$
\frac{\partial \chi(q)}{\partial q}=-\frac{1}{\left\langle n_{A}\right\rangle} \frac{e^{\beta F(q)}}{\int_{q_{A}}^{q_{B}} e^{\beta F(q)} d q} .
$$

We choose

$$
\begin{aligned}
& n_{A}(q)=1-\frac{\int_{q_{A}}^{q} e^{(a-1) \beta F\left(q^{\prime}\right)} d q^{\prime}}{\int_{q_{A}}^{q_{B}} e^{(a-1) \beta F\left(q^{\prime}\right)} d q^{\prime}}, \\
& w(q)=e^{a \beta F(q)}, \\
& \pi(q) \propto e^{(a-1) \beta F(q)},
\end{aligned}
$$

where $a>0$ is a biasing parameter, leading to

$$
k_{A \rightarrow B}=\frac{1}{\left\langle n_{A}\right\rangle} \frac{\left\langle\int_{0}^{\infty} \dot{q}(t) \dot{q}(0) \frac{w[q(t)]}{w[q(0)]} \frac{e^{-\beta F[q(t)]}}{e^{-\beta F[q(0)]}} d t\right\rangle_{\pi}}{\int_{q_{A}}^{q_{B}} e^{\beta F(q)} d q \int_{q_{A}}^{q_{B}} e^{-\beta F(q)} d q} .
$$

Although Eq. (38) can be considered a TST method using a more continuous "detector" function than the noisy $\theta$ function, it can also be viewed as a MD method in which starting configurations are sampled in a more convenient ensemble $\pi(q) \propto w(q) e^{-\beta F(q)}$ and subsequently a weighted velocity autocorrelation is computed. It is important to note that the actual dynamics of the particles is still generated using $\mathrm{MD}$ in the conventional microcanonical ensemble 
without biasing potential. Importance-sampled MD is especially applicable to systems with erratic free-energy landscapes, e.g., multiple barriers of possibly different heights. Note that the expression given by Ruiz-Montero et al. in Ref. 7 reduces to Eq. (38) with $a=2$ when the estimated free energy in their expression is the true free energy.

\section{G. Dynamically corrected transition state theory at nonzero loading}

The extension of dcTST to finite loading is nontrivial. Conventional methods use a hierarchical approach to compute elementary hopping rates $k_{A \rightarrow B}^{\text {iso }}$ between isolated cages $A$ and $B$ for use in a subsequent kMC scheme to obtain diffusion coefficients. However, the fundamental question is whether it is possible to compute an elementary hopping rate $k_{A \rightarrow B}^{\text {iso }}$, in which the contributions of other cages are separated from the contribution of only the cages $A$ and $B$. Let us consider the class of window/cage-type systems (e.g., methane in LTA) where the barriers are entropic in nature. At nonzero loading a molecule hopping from $A$ to $B$ induces a vacancy. While in principle, a particle originating from any of the surrounding cages could fill the vacancy, hierarchical approaches will allow only a molecule from $B$ to return to $A$ (e.g., by blocking all the windows except the window between cages $A$ and $B$ ). The fundamental assumption of kMC (no two jumps can occur at the same time) artificially suppresses these correlated jumps, and we are not aware of a scheme that results in effective kMC hopping rates that regain those correlations. Another way to look at this is that the correlated jumps should be identified as elementary kMC moves.

Beerdsen et $a l^{33}$ proposed a method to compute diffusivity values directly in systems with high free-energy barriers (e.g., cage/window-type zeolites). Here, long time, large distance memory effects are negligible, because once a molecule jumps thermal equilibration takes place and next- nearest cage correlations are rare. It is therefore sufficient to include correlations during the jump across the barrier. Hence, we compute

$$
\begin{aligned}
& D(c)=\frac{1}{6} k_{A \rightarrow B}^{\mathrm{eff}}(c) \lambda^{2}, \\
& k_{A \rightarrow B}^{\mathrm{eff}}(c)=f(c) k_{A \rightarrow B}(c),
\end{aligned}
$$

where $c$ denotes the loading in molecules per unit cell, or $\mathrm{mol} / \mathrm{kg}$. But rather than attempting to compute $k_{A \rightarrow B}(c=0)$ or $k_{A \rightarrow B}^{\text {iso }}$ from a molecular simulation and the correlation factor $f(c)$ from a coarse-grained kinetic Monte Carlo method, Beerdsen et al. compute $k_{A \rightarrow B}^{\text {eff }}(c)$ directly from a molecular simulation where the precise definition of $k_{A \rightarrow B}^{\text {eff }}(c)$ is: $k_{A \rightarrow B}^{\text {eff }}(c)$ is the hopping rate of a single-tagged particle at an average loading $c$ from cage $A$ to cage $B$ under the influence of an external field exerted by the molecular sieve and the other $N-1$ particles. By including the nearest-neighboring cages, all relevant short-time correlations are properly captured, including the dominant short-time backcorrelation effects due to particle-particle interactions. Correlations at much longer times than $1 / k$ are negligible in cage/window- type systems. The computation once again consists of two parts.

- The probability density $P_{\in A}\left(q^{*}\right)$ of finding the system at the top can be computed explicitly by computing freeenergy profiles making use of Eq. (27). During a separate MC simulation in the $N V T$ ensemble at the desired loading we measure the free energy $F(q)$ by using histogram sampling (HS). In the HS method, a histogram is made of the particle positions, mapped on the reaction coordinate. From the histogram a free-energy profile is computed, by using

$$
\beta F(q)=-\ln \langle P(q)\rangle .
$$

At conditions where conventional MC is still feasible, all particles can be considered equivalent and all contributions can be used.

When displacement of particles is impeded by high freeenergy barriers, conventional HS becomes unfeasible. A single, tagged particle can be biased to achieve improved statistics by using importance sampling. As a biasing potential the Widom particle insertion (WPI) profile can be used. WPI uses a probe particle that is inserted at random positions, to measure the energy required for or obtained by insertion of the particle in the system. This energy is mapped onto the reaction coordinate $q$, using

$$
\beta F(q)=-\ln \left\langle e^{-\beta \Delta U}\right\rangle_{N}
$$

to produce a free-energy profile, where $\left\langle e^{-\beta \Delta U}\right\rangle_{N}$ is the average Boltzmann factor over all positions in the slice perpendicular to the reaction coordinate. A "ghost particle" is used as the measuring probe, but the other particles in the system do not feel its presence. At higher loadings, WPI is known to give erroneous results ${ }^{1,33}$ and therefore the WPI method is not used to compute $F(q)$ directly, but rather to estimate the biasing function when needed.

- The particle flux $R(t)$ through the dividing surface can be computed from the fraction of particles starting on the top of the barrier that successfully reach cage $B$. The other particles present in the system influence this fraction. Starting configurations are generated with one particle constrained to the dividing surface and $N-1$ particles moving around (see Figs. 4 and 5). These configurations are then used to compute the particle flux in unconstrained NVE-MD simulations, starting with velocities sampled from a Maxwell-Boltzmann distribution at the desired temperature.

In Fig. 4 an instructive snapshot of methane in LTL-type zeolite at a loading of 3 molecules per unit cell at $300 \mathrm{~K}$ is shown. The free-energy profile consists of maxima, corresponding to geometric constrictions, and minima, corresponding to the apertures. A natural hopping lattice is formed by the one-dimensional sequences of free-energy minima $\left(q_{A}, q_{B}, q_{C}\right.$, etc.). As can be seen from this snapshot, there are strong adsorption sites where the curvature of the zeolite is the highest and commensurate with the shape of the particle. Particles reside in these minima for a long time, before a 


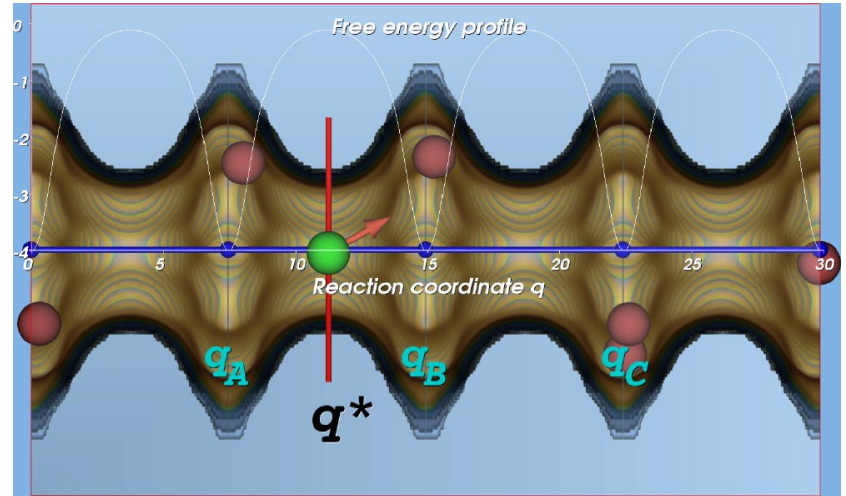

FIG. 4. A typical snapshot of a tagged methane particle (green color) in LTL-type zeolite restrained to the barrier surface $q^{*}$ at an average loading of 3 methane molecules per unit cell (there are two parallel channels per unit cell) at $300 \mathrm{~K}$. Four unit cells of $7.474 \AA$ in length are shown. The constrictions are caused by the 12-T-membered rings, which form free-energy barriers impeding diffusion. The free-energy profile in units of $k_{B} T$ at this average loading is plotted in white, where the reaction coordinate is chosen parallel to the channel direction. If the free-energy barriers are high enough, diffusion can be considered a hopping process from minima to minima $\left(q_{A}\right.$, $q_{B}, q_{C}$, etc)

thermal excitation will eventually give the particles enough mobility to cross the free-energy barrier and proceed to a neighboring lattice site. The latter process is a fast process in comparison to the time a particle spends near the lattice points.

Figure 5 shows a snapshot of ethane at an average loading of 4 molecules/cage at $750 \mathrm{~K}$ in LTA-type zeolite. The lattice, formed by the cage centers, is the three-dimensional cubic lattice. For this snapshot cage $B$ contains more molecules than cage $A$, and the barrier molecule has a high probability of recrossing to cage $A$. The time-dependent transmission coefficient will reach a plateau value $\kappa$. However, because during a successful hop cage $A$ donated a particle, while cage $B$ received an additional particle, there is a slightly higher probability for the particle to return to $A$ on a time scale larger than the thermalization time. However, for our systems this effect is negligible. Note that during the

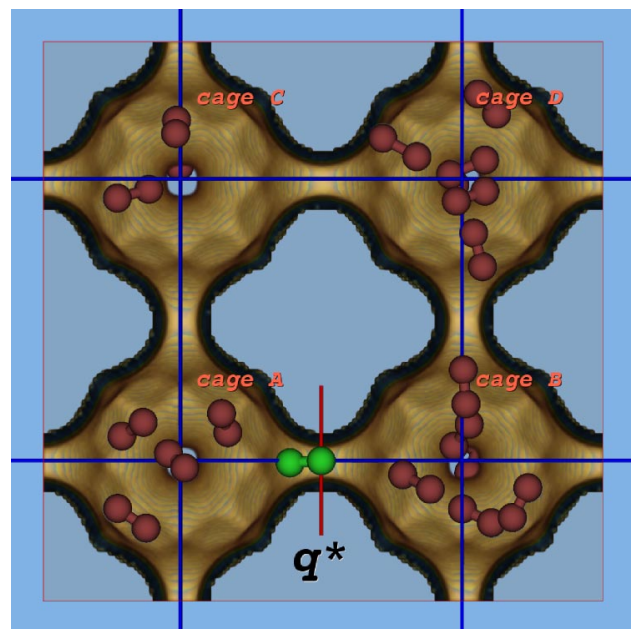

FIG. 5. A typical snapshot of ethane $\left(\mathrm{CH}_{3}-\mathrm{CH}_{3}\right)$ in LTA-type zeolite at an average loading of 4 molecules/cage at $750 \mathrm{~K}$, constraining one tagged molecule at the dividing surface $q^{*}$. The hopping events are coarse grained on a lattice spanned by the cage centers. computation none of the windows is blocked and simultaneous jumps (e.g., from cage $C$ to cage $A$, and cage $D$ to cage $B$ ) are allowed.

The extension of the importance-sampled MD method Eq. (38) to nonzero loading is similar. The method can be summarized as follows:

- The free-energy profiles at the desired average loading are measured as described above.

- The free-energy minima $q_{A}$ and $q_{B}$, and the corresponding hopping lattice are identified.

- A biasing profile $w(q)$ is constructed ranging from $q_{A}$ to $q_{B}$ using Eq. (36).

- Starting configurations are sampled in the interval $q_{A}$ to $q_{B}$ with the bias potential $w(q)$ operating only on the tagged particle, leaving the others $N-1$ free to move (unbiased).

- These starting configurations of $N$ particles are integrated using MD for short times $t_{\max }$, and Eq. (38) is evaluated. The time $t_{\max }$ is chosen such that the integral appearing in Eq. (38) has converged. The trajectories are stopped after $t_{\max }$ time has elapsed or when $q<q_{A}$ or when $q>q_{B}$.

As mentioned, in general, the reaction coordinate $q$ is a function of the configuration of the whole system. For dcTST simulations at a certain loading, we choose the reaction coordinate as the position of one of the atoms of the tagged molecule. ${ }^{33}$ Although it cannot be excluded that better reaction coordinates exist, for physical reasons our choice seems optimal. The diffusion mechanism is divided into two parts. The first is a static term, corresponding to the locations of preferable adsorption sites and estimations of free-energy barriers in between, the latter (or actually the inverse of the transmission coefficient: the recrossing) corresponds to collision frequencies, which generally increase with loading. As such the dcTST method is able to explain different diffusion regimes over loading, and provides insight into the mechanisms behind an increase or decrease in diffusivity with loading. ${ }^{52}$

\section{H. Zeolite descriptions and simulation details}

To test and explore the limits of our dcTST method, we have selected two types of zeolites: the one-dimensional channel LTL-type zeolite structure and three-dimensional cage/window LTA-type zeolite structure. The LTA-type zeolite is selected because diffusion is slow, but just fast enough for the smallest alkanes to allow for a comparison of dcTST with MD. Here, dcTST is expected to work flawlessly. In LTL-type zeolite the diffusion is relatively fast, the freeenergy barriers low, and the system is close to the limits of TST, i.e., in this system it is more difficult to envision a clear separation of time scales. Moreover, the system is one dimensional and correlations between diffusing particles are even higher.

LTL-type zeolites are used industrially for the aromatization of alkanes. The structure ${ }^{53}$ has space group $P_{6} / \mathrm{mmm}$ 
with $a=1.84, b=1.84, c=0.752 \mathrm{~nm}$, and $\alpha=\beta=90^{\circ}, \quad \gamma$ $=120^{\circ}$. For computational efficiency the unit cell is converted to a rectangular cell. LTL-type zeolite provides a pore system having cancrinite cages (11-hedra) alternating with hexagonal prisms (8-hedra) stacked in columns parallel to the $c$ axis. The channels thus formed have nearly planar 12membered rings with a free diameter of approximately $0.71 \mathrm{~nm}$ and expansions of approximately $0.126 \mathrm{~nm}$ (see Fig. 4).

The single largest use of zeolites is the use of LTA-type zeolites for laundry detergents. LTA-type zeolite is also used for separations of small molecules from air by exploiting different polarities of molecules, and for bulk separations of linear and branched alkanes. The LTA-type structure ${ }^{54}$ has a cubic space group $F m \overline{3} c$ with $a=b=c=2.4555 \mathrm{~nm}$, and $\alpha$ $=\beta=\gamma=90^{\circ}$. The crystallographic unit cell consists of eight large spherical cages (named $\alpha$ cages) of approximately $1.12-\mathrm{nm}$ interconnected via windows of about 0.41-nm diameter (see Fig. 5).

In addition to the relevant cages and channels there are also topologically disconnected pockets. A methane molecule does fit at that position, but it is not accessible from the main cages and channels. Both LTA (sodalite cages), as well as LTL, have disconnected pockets. To obtain correct results in MC simulations it is necessary to ensure that molecules will not be inserted into inaccessible pockets for adsorbing molecules.

We have summarized the details of our periodic simulation boxes in Table III. Simulation of one-dimensional channels requires special attention. Here, diffusion results are very much dependent on the length of the channel, and surprisingly long channels are needed to reliably extrapolate to macroscopic diffusion coefficients. ${ }^{55}$

\section{RESULTS}

\section{A. Infinite dilution}

\section{Methane, ethane, and propane in LTL zeolite}

Figure 6 shows the free-energy profiles $F(q)$ of methane along the channel direction. Two free-energy minima are separated by a 12-ring forming the free-energy barrier at $q^{*}$. For this system and the chosen reaction coordinate, the transmission coefficient is nearly equal to 1 , and the dcTST diffusion can be directly computed using Eqs. (20) and (28). The free-energy barrier ranges from about $10 k_{B} T$ at $100 \mathrm{~K}$ to less than $3 k_{B} T$ at $1000 \mathrm{~K}$.

The transmission coefficient can only be assumed equal to one for a single, spherical particle, provided the exact barrier is known, and only at infinite dilution. Even for methane in LTL the position of the barrier is only known approximately because of the atomic structure of the window, although the value is very close to 1 using the window as the dividing surface. In Fig. 7 the transmission coefficient $\kappa(t)$ for propane using the second/middle bead as the reaction coordinate is shown as a function of time for various temperatures. The starting configurations are sampled using a Monte Carlo scheme at the desired temperature constraining the reaction coordinate (here: the middle bead) to the dividing surface. This distribution of configurations is temperature
TABLE III. Simulation details for the LTL- and LTA-type zeolites. Crystallographic positions are taken from Refs. 53 and 54.

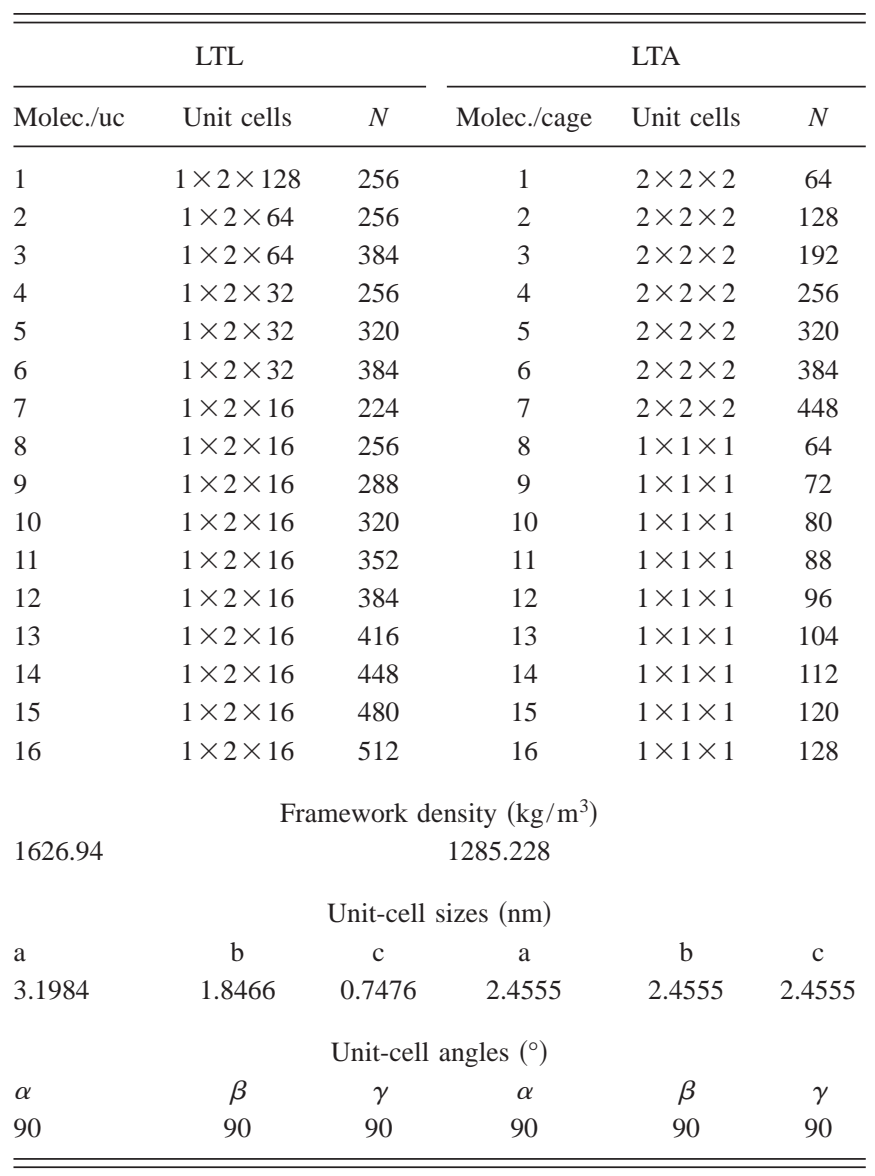

dependent, and also the transmission coefficient is temperature dependent. In general, the transmission coefficient increases with temperature, because a higher fraction of configurations has sufficient kinetic energy to overcome the freeenergy part resulting from a nonoptimal reaction coordinate and dividing surface choice. The transmission coefficient starts at one (by definition, because a particle cannot revert its velocity within a single integration step), and slowly converges to a limiting plateau value at about $15 \mathrm{ps}$. It is this

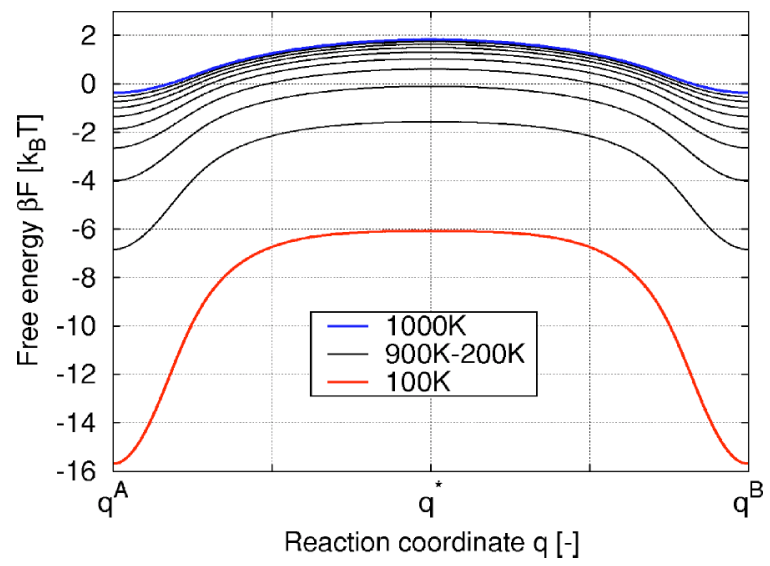

FIG. 6. Free-energy profiles $F(q)$ of methane in LTL at various temperatures $(100-1000 \mathrm{~K}$ in steps of $100 \mathrm{~K})$ and infinite dilution. The reaction coordinate is chosen along the $z$-channel direction (lines from top to bottom in order of the legend). 


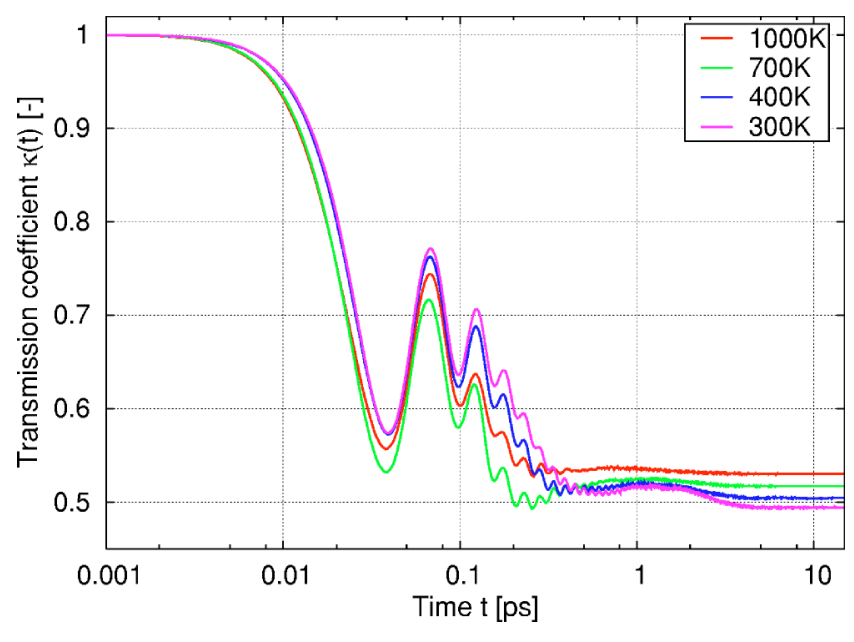

FIG. 7. Transmission coefficient $\kappa(t)$ for propane in LTL-type zeolite at infinite dilution using the second/middle bead as a function of time for various temperatures.

plateau value $\kappa$ that is of interest, signaling all short-time recrossing are eliminated. The intermediate oscillatory behavior is caused by the bond springs within the molecule itself. From the point of view of the reaction coordinate (the position of the second/middle bead), the other beads connected to the middle bead with springs are just an external field in addition to the zeolite.

The transmission rates can be computed from the freeenergy profiles using Eqs. (28) and (30) and transmission coefficients, and then converted to diffusion coefficients using Eq. (20). The methane, ethane, and propane diffusivities computed from dcTST are compared to reference MD simulations in Fig. 8. The MD simulations were performed in the NVE and NVT ensembles (NHC thermostat) with 128 noninteracting particles. For methane, up to $450 \mathrm{~K}$ (barriers higher than $3.5 k_{B} T$ ) dcTST gives equivalent results to MD, but at higher temperatures the methods diverge. For ethane, the methods diverge at $550 \mathrm{~K}$, while for propane both methods overlap. When the methods diverge, the free-energy barriers become too low for TST to be valid, because there is no longer a clear separation of time scales. The methane molecules do not equilibrate properly leading to enhanced diffu-

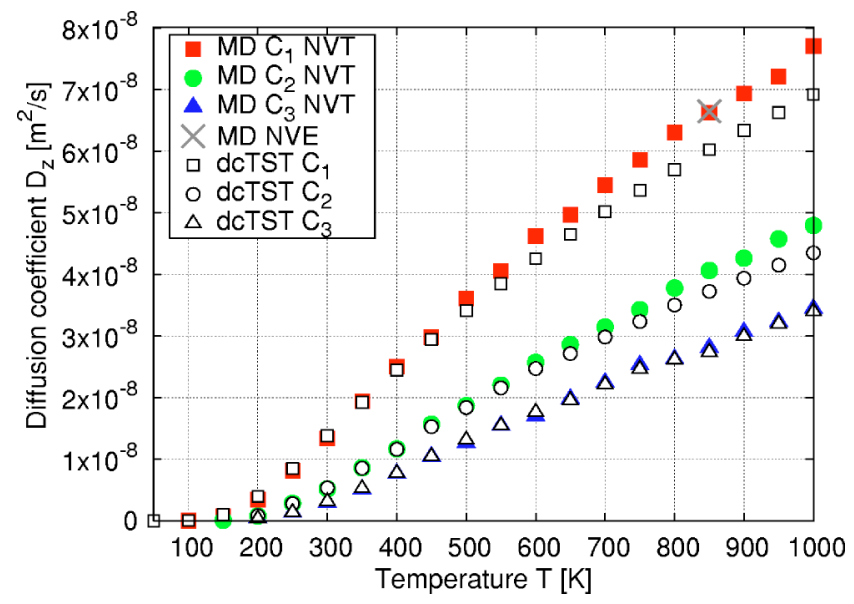

FIG. 8. Diffusion of methane, ethane, and propane in LTL-type zeolite at infinite dilution computed by dcTST and MD.

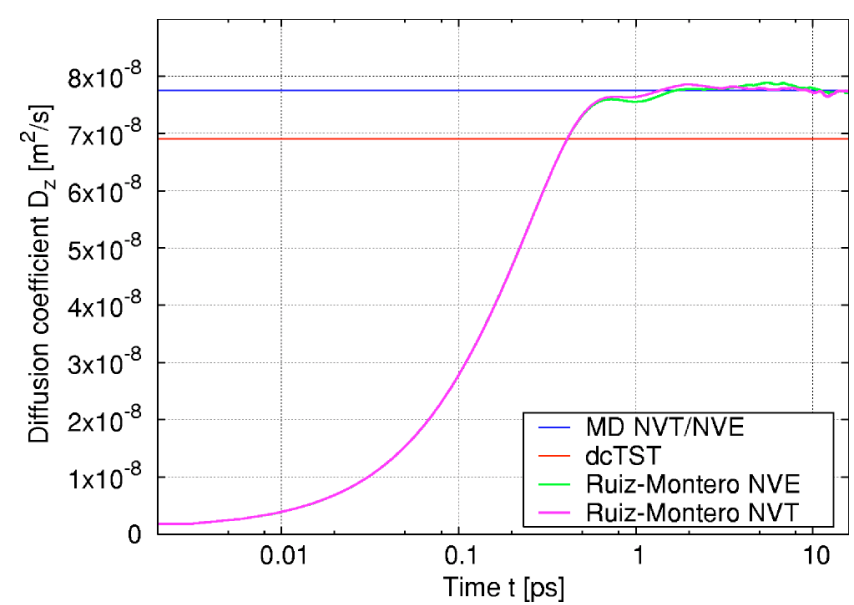

FIG. 9. Diffusion of methane at $1000 \mathrm{~K}$ in LTL-type zeolite at infinite dilution computed by dcTST and MD compared to the method of RuizMontero et al.

sion due to kinetic correlations, i.e., the increased probability of particles to continue in their current direction.

There are several ways to include kinetic correlations, amongst them are the dynamical corrections as formulated by Voter and Doll ${ }^{56}$ using multistate systems and the recently proposed method of Ruiz-Montero et al. [Eq. (38) with $a=2]$. $^{7}$ The method of Voter and Doll would extend the twostate system $A$ and $B$, to a multistate system $A, B, C, D, \ldots$, by computing the hopping rates $k_{i j}$, dynamical corrections $\kappa_{i j}$, and lattice distances $\lambda_{i j}$ between state $i$ and $j$. Note that the dynamical correction in this formulation is not restricted between 0 and 1 , but can potentially increase beyond unity when kinetic correlations are abundant. However, at the highest temperatures reported here, the time scales of thermalization and $k^{-1}$ become inseparable, so we pursue here the alternative route of Ruiz-Montero et al. Figure 9 shows the method of Ruiz-Montero et al. $(a=2)$ at $1000 \mathrm{~K}$ for methane in LTL-type zeolite. The Eq. (38) is reaching a plateau value in time, equal to the MD results. These results differ from the dcTST value due to presence of kinetic directional correlations, which are included using (importancesampled) MD methods. However, computing Eq. (38) is very time consuming and the dcTST method is preferable for cage/window-type systems with higher free-energy barriers.

\section{Methane in LTA-type zeolite}

At infinite dilution, the barrier for diffusion of methane in LTA-type zeolite at $300 \mathrm{~K}$ is much higher than in LTLtype zeolite. The barrier is approximately $8.5 k_{B} T$ and sharply peaked. In Fig. 10 we compare various biasing functions for the importance-sampled MD method. A biasing weighting function of

$$
w(q)=\frac{e^{a \beta F(q)}}{\int e^{a \beta F(q)} d q},
$$

using $a=1$ flattens the free-energy landscape and the initial configurations are sampled uniformly. However, for diffusion only the configurations in the barrier region yield relevant information, and with $a \gg 1$ as a biasing function the con- 


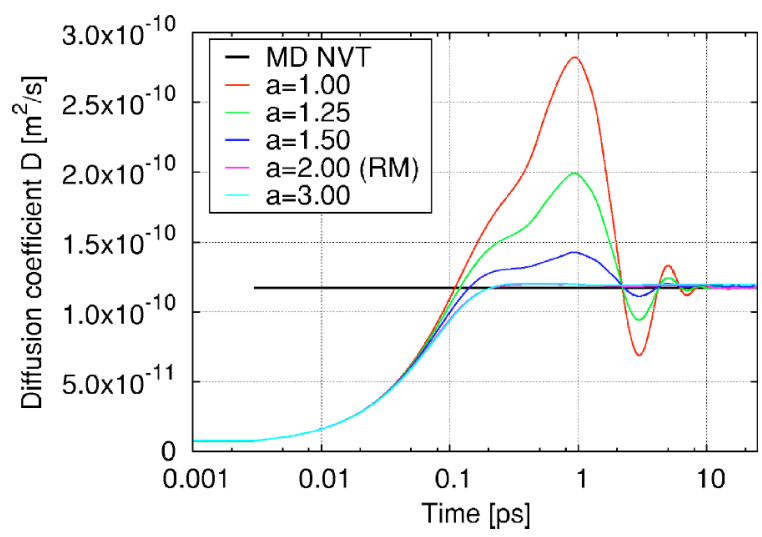

FIG. 10. Diffusion of methane over a high free-energy barrier in LTA-type zeolite at $300 \mathrm{~K}$ and infinite dilution using various biasing functions [Eq. (43)]. The method of Ruiz-Montero (RM) et al. uses $a=2$.

figurations are indeed restricted to the barrier regions, and all trajectories contribute significantly to the diffusion coefficient. The results show that for sharply peaked barriers a high biasing function achieves fast convergence. For these barriers the Bennett-Chandler method works well, because diffusive behavior is negligible and dynamic corrections are easily evaluated. However, the importance-sampled MD method is also applicable in the diffusive regime where $\kappa \ll 1$.

\section{Ethane in LTA-type zeolite}

Ethane molecules in LTA perform jumps on a simple cubic lattice. It was found that self-diffusion decreases with increasing temperature at low temperatures. ${ }^{24}$ At low temperatures the molecules become less confined in the windows as temperature increases. Heating the system, ethane moves away from the windows, which increases the entropic barrier for cage-to-cage motion. Figure 11 shows that the behavior found by Schüring et al. ${ }^{24}$ is strongly dependent on the parameter set (Tables I and II). The parameter set of Dubbeldam et al. $^{39}$ does not show a decrease with increasing temperature although for both parameter sets the local acti-

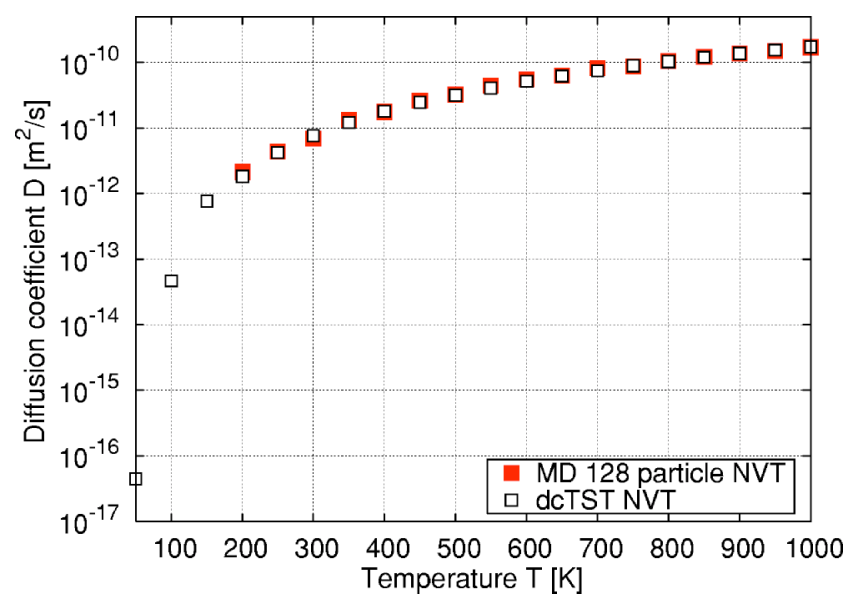

FIG. 11. Self-diffusion of ethane at infinite dilution in LTA-type silica using the parameter set of Dubbeldam et al. The infinite dilution case using MD is simulated by switching the ethane intermolecular forces off, i.e., the particles do not interact (except through the thermostat in NHC-NVT simulations).

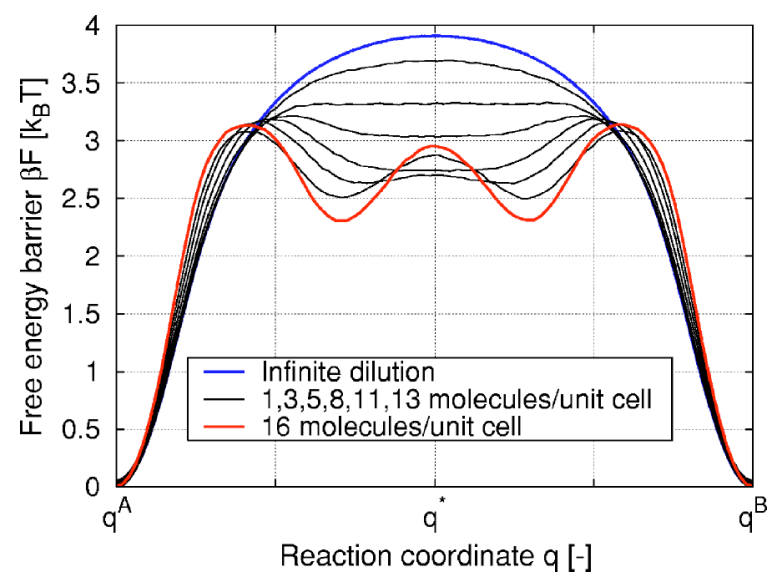

FIG. 12. Free-energy profiles $F(q)$ at $300 \mathrm{~K}$ of methane in LTL at various loadings (infinite dilution, 1, 3, 5, 8, 11, 13, and 16 molecules per unit cell). The reaction coordinate is chosen along the $z$-channel direction (lines from top to bottom in order of the legend).

vation energy depends on temperature. The size parameter used by Schüring et al. is so small that ethane at low temperature is found in the windows itself, and heating shifts the adsorption sites to just in front of the windows. In contrast, the set of Dubbeldam et al. has a larger size parameter for ethane and also at low temperature the adsorption sites are always in front of the window, reducing the behavior found by Schüring et al. However, the phenomenom is likely to be generic and present in cage/window-type systems and applicable to small molecules. It shows how much the actual adsorption sites can depend on temperature and simulation parameters.

\section{B. Nonzero loading}

\section{Methane, ethane, propane in LTL-type zeolite}

The free-energy barriers for various loadings of methane in LTL-type zeolite are plotted in Fig. 12. In comparison to the infinite dilution case, the free-energy barrier initially decreases. Adding particles to the system induces an effectively "smoother" channel. For increasing loadings the top of the barrier flattens and eventually transforms into a barrier region with two local free-energy minima.

Figure 13 shows the diffusion behavior as a function of loading in LTL-type zeolite for methane, ethane, and propane at $300 \mathrm{~K}$. The TST diffusivities based on the free-energy profiles (e.g., for methane shown in Fig. 12) increase, while the dcTST values decrease and are equal to the conventional MD results. Clearly the transmission coefficient not only quantitatively, but also qualitatively, correct the TST results. The good agreement between dcTST and MD for LTL-type zeolites is encouraging. The diffusion of alkanes in LTL-type zeolite is quite fast, and the fact that our dcTST method also works for such low free-energy barriers as in LTL-type zeolite is surprising.

Although this region of diffusion is fully accessible to conventional MD, the dcTST method has a very important advantage: it enables us to explain the qualitative behavior of diffusion in terms of free-energy difference and transmission coefficients. For example, initially the diffusion of methane in LTL-type zeolite does not change much with loading. The 


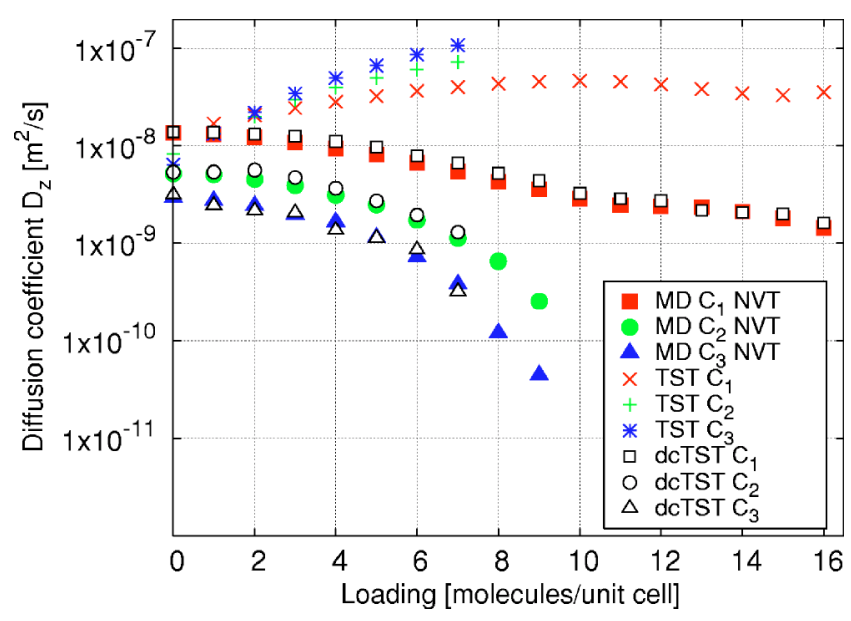

FIG. 13. Diffusion of methane, ethane, and propane at $300 \mathrm{~K}$ as a function of loading in LTL-type zeolite computed by TST, dcTST, and MD.

MD results would suggest the fundamental reason could be that particles hardly notice each other at these lower loadings. However, the picture painted by the dcTST is quite different. There are two effects: (1) the free-energy barrier decreases with loading (which means an increase in diffusion) rendering the channel environment more "uniform," and (2) as loading increases the transmission coefficient decreases due to an increased collision frequency. At low loading the two effects almost counterbalance each other, but at higher loadings the rapid increase in collision frequency dominates.

An important observation made by Beerdsen et al. ${ }^{52}$ is that the appearance of the two local minima on top of the free-energy barrier at around 11 molecules per unit cell for methane causes an inflection at the corresponding loading in the diffusion curves. This inflection is similarly found in the adsorption isotherms, and both are related to a change in packing. They show that the dcTST method can generally be used to qualitatively explain the self and collective diffusion behaviors of a molecule/zeolite combination as a function of loading, by carefully analyzing the change in free-energy (packing effects).

The appearance of extra adsorption sites with loading, and the change of packing, shows that the choice of the random-walk lattice cannot solely be based on a lattice of adsorption sites at low loading. The adsorption-site lattice needed to describe diffusion over adsorption sites depends on the zeolite, the guest, the temperature, and on the loading of the zeolite. In fact, for every zeolite, guest, temperature, and loading the lattice should be reconstructed. However, for very slow diffusion in cage/window-type zeolites the ratedetermining step is the cage-to-cage motion and all the details of intracage diffusion are present in the free-energy profile. For very fast diffusion the construction of a lattice based on adsorption sites does not make sense either as there is too much correlation present between the hops at such a lattice, i.e., the separation of time scales vanishes and the description as "hopping" breaks down. The natural lattice to use would be an effective lattice of cage-center to cage-center.

For zeolites accessible to conventional MD the freeenergy can be obtained using either $\mathrm{MC}$ or $\mathrm{MD}$, and we

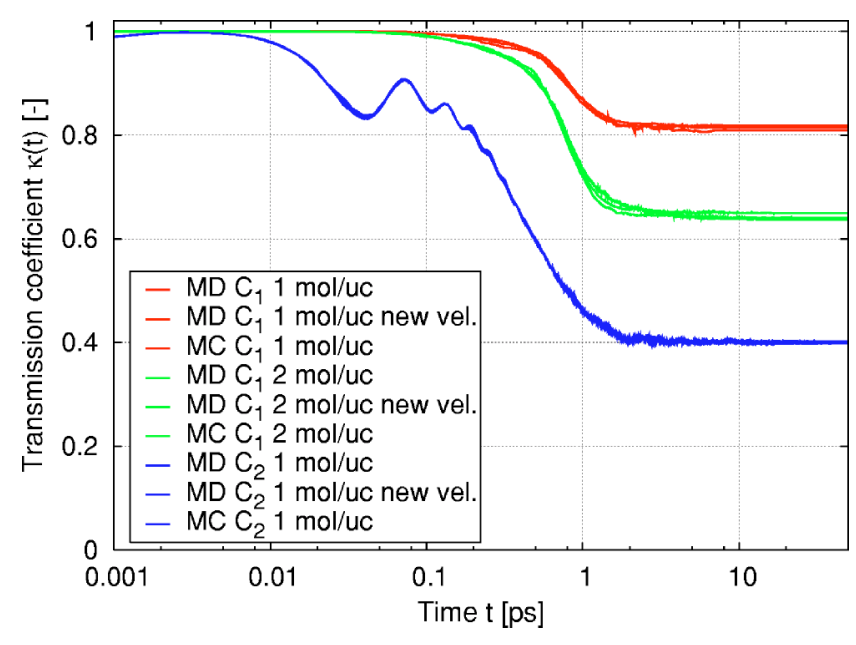

FIG. 14. Transmission coefficient $\kappa(t)$ at $300 \mathrm{~K}$ for methane and ethane in LTL-type zeolite using the first bead as a function of time for various loadings. The starting configurations are sampled with MC or with MD, where the velocities are either taken from the trajectory or newly resampled from the Maxwell-Boltzmann distribution. The differences are negligible.

found no differences between both methods. In contrast, the sampling of configurations with a tagged particle restricted to the dividing barrier surface requires more thought. In Fig. 14 we show results for the transmission coefficients using MC and MD. The MD results are splitted into two types: (1) both the positions and velocities are stored, and (2) only the positions are taken from MD trajectories, the velocities are resampled from a Maxwell-Boltzmann distribution. As can be seen, reinitializing the velocities holds equal results, and one can conclude from the result that the velocities in this system are indeed Maxwell-Boltzmann distributed, even on top of the barrier. For systems with higher free-energy barriers configurations are adequately sampled with biased $\mathrm{MC}$, while conventional MD would become impossible.

\section{Methane, ethane in LTA-type zeolite}

Figure 15 shows the free-energy profiles at $600 \mathrm{~K}$ for methane in LTA-type zeolite for various loadings. Relative to the infinite dilution case, the addition of particles to the cages leads to an increase of the free-energy inside the cage, while the free-energy at the barrier remains unchanged up to inter-

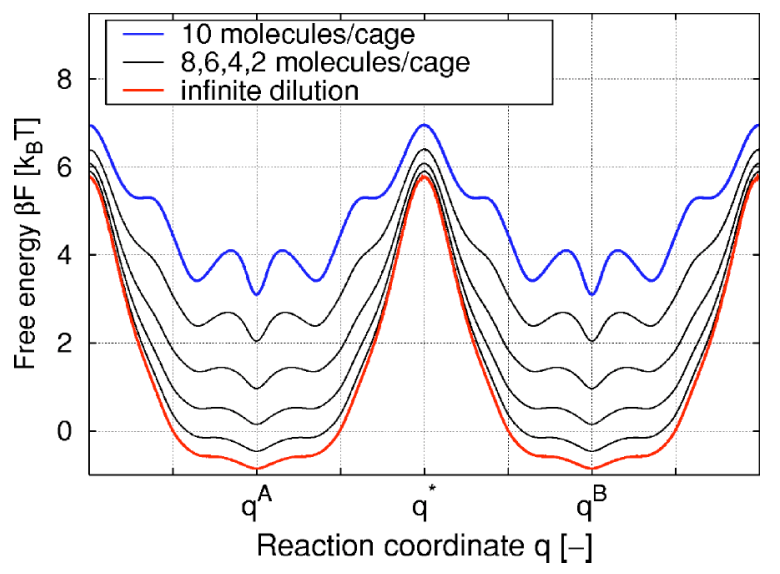

FIG. 15. Free-energy profiles of methane in LTA-type zeolite at $600 \mathrm{~K}$ for various loadings $(10,8,6,4$, and 2 molecules/cage, and infinite dilution). 

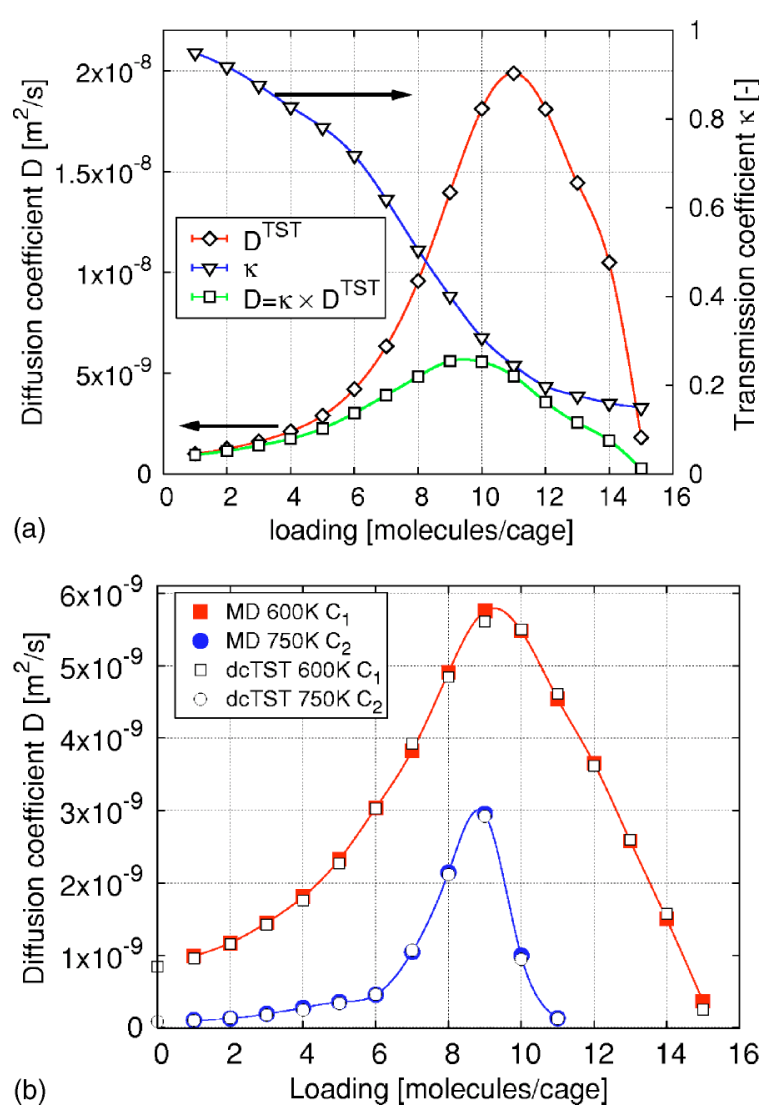

FIG. 16. dcTST and MD in LTA-type zeolite (a) the TST and dcTST diffusivities for methane at $600 \mathrm{~K}$ as a function of loading using the left axis, and the tranmission coefficient $\kappa$ using the right axes, (b) diffusion of methane and ethane in LTA-type zeolite, as a function of loading, at 600 and $750 \mathrm{~K}$, respectively.

mediate loading. The inner-cage surface of LTA-type zeolite is adsorbophylic (wetting regime), and upon increased adsorption favorable adsorbate-adsorbent interactions are being replaced by less favorable interaction with other particles.

Figure 16(a) shows the individual components of the diffusion process, $D^{\mathrm{TST}}$ and $\kappa$ as a function of loading for methane in LTA. Although the transmission coefficient shows a monotonic decrease with density, the diffusion coefficient goes through a maximum. The driving force behind the initial increase in diffusion is a loss of guest-host attraction inside the cages. Eventually, the free-energy barrier increases again, due to packing and free-volume effects, causing a decrease of the diffusion coefficient. While the transmission coefficient only slightly changes the qualitative behavior of the diffusion as a function of loading, it has a profound quantitative influence. We show the diffusion in LTA of methane at $600 \mathrm{~K}$ and ethane at $750 \mathrm{~K}$ using both MD and extended dcTST in Fig. 16(b). Our extended dcTST method and MD again agree quantitatively.

\section{Methane/ethane-mixture in LTA-type zeolite}

In Fig. 17 we plotted the results for a 50\%-50\% mixture of methane and ethane in LTA-type zeolite, as a function of loading at $300 \mathrm{~K}$. For each of the components the freeenergy and transmission coefficients are computed. For the $\kappa$ - computation a single molecule of the component is re-

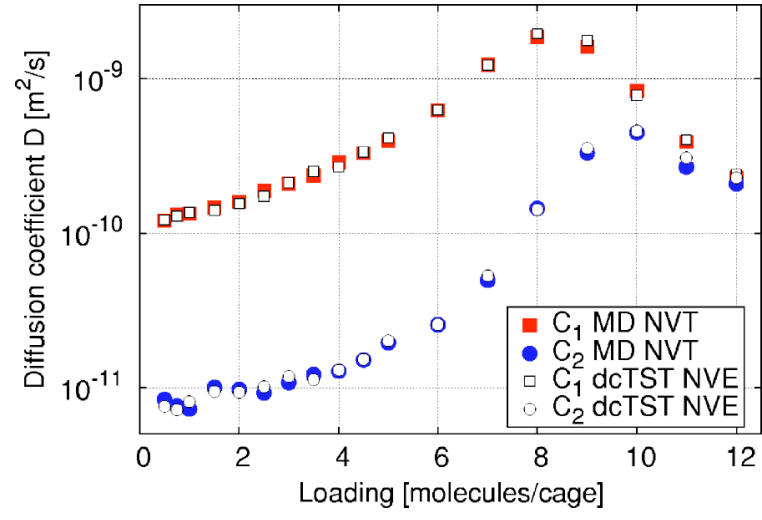

FIG. 17. Self-diffusion in a 50\%-50\% mixture of methane and ethane in LTA-type zeolite, as a function of loading at $300 \mathrm{~K}$.

stricted to the barrier, while the other molecules of the same component, and all molecules of the other component are free to move. Again, our extended dcTST method and MD agree quantitatively.

\section{DISCUSSION}

We have shown that our method is applicable to simulating self-diffusion in any elementary topology. The extension of Eq. (17) to more complex structures and lattices is a geometric exercise to be published elsewhere. ${ }^{57}$ The connection between the random-walk lattice and the zeolite structure is found by an analysis of the free-energy profiles. The diffusion of a tagged molecule is computed over that typical length scale given by the smallest repeating zeolite structure, i.e., from the center of cage $A$ to the center of cage $B$. One automatically averages over all adsorption sites in the cage, irrespective whether the adsorption sites are strong or weak, or even ill defined, i.e., for purely entropic barriers. For most lattices the equivalent of Eq. (17) has been worked out. Since the lattice is not based on specific adsorption sites, often the same lattice can be used for all temperatures and loadings, although sometimes at high loadings new barriers may be formed.

Tunca and Ford ${ }^{29-31}$ computed elementary hopping rates using multidimensional TST for use in a subsequent coarsegrained kinetic Monte Carlo (kMC) scheme. Besides the various approximations to make the computation tractable, this approach relies on the computation of an elementary hopping rate. The fundamental question about hierarchical approaches is "is it possible to compute an elementary hopping rate?" In our calculations, we have observed that to obtain agreement with MD results, one cannot limit the freeenergy calculation to the two cages $A$ and $B$ for which the hopping is computed. It is essential to average over fluctuations in the number of particles in the neighboring cages. ${ }^{33}$ By "closing off" cages, the system is intrusively changed and we are not aware of any other scheme that can separate the contributions of other cages from the contribution of only the cages $A$ and $B$. The omitted correlations are not the same as those regained by a kMC simulation later and therefore further corrections are needed to obtain results in exact agreement with MD. 
We explicitly avoid the use of kMC and compute the self-diffusion coefficient directly. The diffusion constant we compute is the self-diffusion of a tagged molecule traveling from cage $A$ to cage $B$ considering all other particles as an external field. The external field is maintained by a MC NVT simulation (fixed total number of particles, volume, and temperature) of spectator molecules in the "background." By using a MC approach that includes translational, orientational, and regrow moves, we average over cage distributions, positions and orientations of neighboring molecules. This renders it unnecessary to sample the complete phase space by integrating over all particle positions and orientations, weighed with the correct Boltzmann weight. In addition to being computationally much cheaper, it also allows for the use of advanced simulation techniques such as CBMC, which speeds up simulations of longer molecules by orders of magnitude. Longer molecules are efficiently handled and likewise, diffusion in mixtures can easily be computed; all particles are considered part of the external field, irrespective of the type of particle. The LTA-type system used here is a cation-free version of the commonly used LTA 5A zeolite ( $4 \mathrm{Na}^{+}$and $4 \mathrm{Ca}^{+}$per cage). A quantitative comparison with pulsed field gradient-nuclear magnetic resonance (PFG-NMR) experimental results requires including the ions in the simulations. Beerdsen et al. ${ }^{58}$ and Calero et $a l .{ }^{59}$ have extended the united-atom model with cations, and our dcTST method already includes the necessary tools.

The diffusion behavior of ethane in LTA as a function of temperature has been well studied. In contrast to a previous study of Schüring et al., ${ }^{24,43}$ we found that ethane molecules in LTA-type zeolite perform hops on a regular cubic lattice, even when we used the smaller size parameters of Schüring et al. However, these authors computed $k_{A B}$ from MD using the number of cage visits divided by the MD time. Such an approach overestimates the actual self-diffusivity by nearly an order of magnitude for ethane in LTA-type zeolite at $100 \mathrm{~K}$. Using the center of mass of ethane as the order parameter, they overestimate the rate, because a molecule coming from $A$ will show diffusive behavior in the barrier region and change cage many times before equilibrating in $A$ (recrossing) or $B$ (transmission). Only the successful transmission should be counted and Schüring et al. found that the correlation factor $f$ [Eq. (19)] computed using a molecular simulation approximately corrects for this. Our results show that a proper computation of the effective rate constant including the transmission coefficient leads to exact agreement between dcTST and MD. We stress that $\kappa$ and $f$ are different concepts. The similarity in behavior for this specific system originates from the fact that $\kappa$ is dominated by backcorrelations, and for a cubic lattice Eq. (19) computes the same. However, the computation of $f$ using Eq. (19) is limited to the MD time scale.

We would like to comment on the use of flexible zeolites with regard to our dcTST method. Although for computational reasons we kept the zeolite rigid, our method is fully applicable to flexible zeolites. In Ref. 37 it was found that self-diffusion coefficients for methane in LTA-type zeolite obtained with flexible and with rigid lattices are practically the same. In Fig. 18 we show the self-diffusivity of ethane

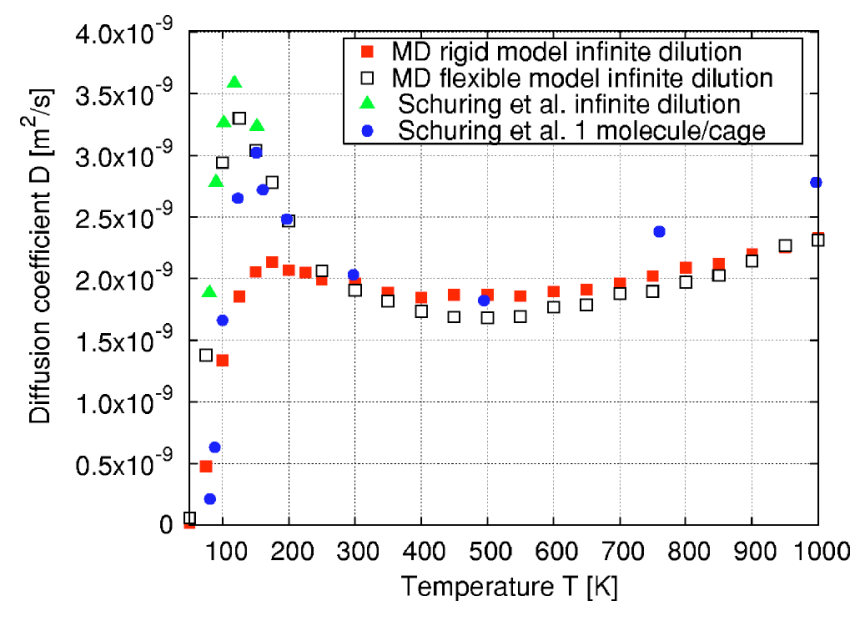

FIG. 18. Self-diffusivity of ethane using the LJ parameters of Table II, comparing the results of a rigid zeolite to the flexible model of Demontis et al. (Refs. 60 and 61). Error bars are smaller or comparable to the symbol size. For comparison we have added the results of Schüring et al. (Ref. 43) for the infinite dilution case, and for 1 molecule/cage.

using the Lennard-Jones (LJ) parameters of Table II and compare the results of a rigid zeolite to the flexible model of Demontis et al. ${ }^{60,61}$ Error bars are smaller or comparable to the symbol size. We have added the results of Schüring et $a l .{ }^{43}$ for the infinite dilution case, and for 1 molecule/cage. The data of Schüring et al. compare well and are consistent with our simulations. An important observation is that the differences between flexible and rigid LTA-type zeolites for ethane are significant and temperature dependent. In the lowtemperature region the ethane molecule is tightly confined in the window itself, while at higher temperature the molecule is less tightly confined and located just in front of the window. The method proposed in this paper would allow a detailed investigation of the effect of framework flexibility on slow-diffusing molecules.

\section{CONCLUSIONS}

Our method applies dcTST at nonzero loadings without introducing assumptions not already present in traditional TST methods. It can be used to explain diffusion behavior as a function of loading in any system with enough energy dissipation between hops, so that random-walk theory (the assumption of equilibration between two subsequent jumps) and TST are valid, as we showed here for small alkanes in LTL- and LTA-type zeolites. The method gives results in exact agreement with MD, but is also applicable in the regime of very slow diffusion where MD cannot be used. This extends the range of accessible time scales significantly beyond currently available methods. Furthermore, the method enables us to express loading effects in terms of free energy differences. It can be used in any lattice and for any adsorbate, and also for mixtures.

\section{ACKNOWLEDGMENTS}

We thank D. Frenkel, T. S. van Erp, and S. Calero, for their comments on the manuscript, the Netherlands Research 
Council (CW) and the Deutsche Forschungsgemeinschaft (DFG, priority program SPP 1155) for financial support, and NWO/NCF for computational resources.

${ }^{1}$ D. Frenkel and B. Smit, Understanding Molecular Simulation, 2nd ed. (Academic, London, UK, 2002).

${ }^{2}$ M. P. Allen and D. J. Tildesley, Computer Simulation of Liquids (Clarendon, Oxford, 1987).

${ }^{3}$ J. M. Thomas, Sci. Am. 266, 112 (1992).

${ }^{4}$ A. F. Voter, F. Montalenti, and T. C. Germann, Annu. Rev. Mater. Res. 32, 321 (2002).

${ }^{5}$ C. H. Bennett, in Diffusion in Solids: Recent Developments, edited by A. Nowick and J. Burton (Academic, New York, 1975), pp. 73-113.

${ }^{6}$ D. Chandler, J. Chem. Phys. 68, 2959 (1978).

${ }^{7}$ M. J. Ruiz-Montero, D. Frenkel, and J. J. Brey, Mol. Phys. 90, 925 (1996).

${ }^{8}$ P. G. Bolhuis, C. Dellago, and D. Chandler, Faraday Discuss. 110, 421 (1998).

${ }^{9}$ T. S. van Erp, D. Moroni, and P. G. Bolhuis, J. Chem. Phys. 118, 7762 (2003).

${ }^{10}$ D. Moroni, P. G. Bolhuis, and T. S. van Erp, J. Chem. Phys. 120, 4055 (2004).

${ }^{11}$ A. F. Voter, Phys. Rev. Lett. 78, 3908 (1997).

${ }^{12}$ A. F. Voter, Phys. Rev. B 57, 013985 (1998).

${ }^{13}$ M. R. Sorensen and A. F. Voter, J. Chem. Phys. 112, 9599 (2000).

${ }^{14}$ G. Henkelman and H. Jónsson, J. Chem. Phys. 115, 9657 (2001).

${ }^{15}$ R. L. June, A. T. Bell, and D. N. Theodorou, J. Phys. Chem. 95, 8866 (1991).

${ }^{16}$ R. Q. Snurr, A. T. Bell, and D. N. Theodorou, J. Phys. Chem. 98, 5111 (1994).

${ }^{17}$ R. Q. Snurr, A. T. Bell, and D. N. Theodorou, J. Phys. Chem. 98, 11948 (1994).

${ }^{18}$ E. J. Maginn, A. T. Bell, and D. N. Theodorou, J. Phys. Chem. 100, 7155 (1996).

${ }^{19}$ F. Jousse and S. M. Auerbach, J. Chem. Phys. 107, 9629 (1997).

${ }^{20}$ T. R. Forester and W. Smith, J. Chem. Soc., Faraday Trans. 93, 3249 (1997).

${ }^{21}$ T. Mosell, G. Schrimpf, and J. Brickmann, J. Phys. Chem. B 101, 9476 (1997)

${ }^{22}$ T. Mosell, G. Schrimpf, and J. Brickmann, J. Phys. Chem. B 101, 9485 (1997)

${ }^{23}$ P. K. Ghorai, S. Yashonath, and R. M. Lynden-Bell, Mol. Phys. 100, 641 (2002).

${ }^{24}$ A. Schüring, S. M. Auerbach, S. Fritzsche, and R. Haberlandt, J. Chem. Phys. 116, 10890 (2002)

${ }^{25}$ D. Dubbeldam, S. Calero, T. L. M. Maesen, and B. Smit, Phys. Rev. Lett. 90, 245901 (2003).

${ }^{26}$ D. Dubbeldam and B. Smit, J. Phys. Chem. B 107, 12138 (2003).

${ }^{27}$ B. Smit and J. I. Siepmann, J. Phys. Chem. 98, 8442 (1994).

${ }^{28}$ M. Schenk, S. Calero, T. L. M. Maesen, L. L. van Benthem, M. G. Verbeek, and B. Smit, Angew. Chem., Int. Ed. 41, 2500 (2002).

${ }^{29}$ C. Tunca and D. M. Ford, J. Chem. Phys. 111, 2751 (1999).

${ }^{30}$ C. Tunca and D. M. Ford, J. Phys. Chem. B 106, 10982 (2002).
${ }^{31}$ C. Tunca and D. M. Ford, Chem. Eng. Sci. 58, 3373 (2003).

${ }^{32}$ S. M. Auerbach, Int. Rev. Phys. Chem. 19, 155 (2000).

${ }^{33}$ E. Beerdsen, B. Smit, and D. Dubbeldam, Phys. Rev. Lett. 93, 248301 (2004).

${ }^{34}$ J. P. Ryckaert and A. Bellemans, Faraday Discuss. Chem. Soc. 66, 95 (1978).

${ }^{35}$ M. D. Macedonia and E. J. Maginn, Mol. Phys. 96, 1375 (1999).

${ }^{36}$ T. J. H. Vlugt and M. Schenk, J. Phys. Chem. B 106, 12757 (2002).

${ }^{37}$ S. Fritzsche, M. Wolfsberg, R. Haberlandt, P. Demontis, G. B. Suffritti, and A. Tilocca, Chem. Phys. Lett. 296, 253 (1998).

${ }^{38}$ A. G. Bezus, A. V. Kiselev, A. A. Lopatkin, and P. Q. J. Du, J. Chem. Soc., Faraday Trans. 2 74, 367 (1978).

${ }^{39}$ D. Dubbeldam, S. Calero, T. J. H. Vlugt, R. Krishna, T. L. M. Maesen, and B. Smit, J. Phys. Chem. B 108, 12301 (2004).

${ }^{40}$ D. Dubbeldam, S. Calero, T. J. H. Vlugt, R. Krishna, T. L. M. Maesen, E. Beerdsen, and B. Smit, Phys. Rev. Lett. 93, 088302 (2004).

${ }^{41}$ C. Shang-Shan, H. Jobic, M. Plazanet, and D. Sholl, Chem. Phys. Lett. (to be published).

${ }^{42}$ T. J. H. Vlugt, R. Krishna, and B. Smit, J. Phys. Chem. B 103, 1102 (1999).

${ }^{43}$ A. Schüring, S. M. Auerbach, S. Fritzsche, and R. Haberlandt, in Random Walk Treatment of Dumb-Bell Molecules in an LTA Zeolite and in Chabazite, Proceedings of the 14th International Zeolite Conference, April 2004, Cape Town, South Africa, edited by E. van Steen, L. Callanan, and M. Claeys (Elsevier, Cape Town, 2004), pp. 2110-2117.

${ }^{44}$ D. C. Rapaport, The Art of Molecular Dynamics Simulation, 2 nd ed. (Cambridge University Press, Cambridge, 2004).

${ }^{45}$ K. Kremer and G. S. Grest, J. Chem. Phys. 92, 5057 (1990).

${ }^{46}$ G. J. Martyna, M. Tuckerman, D. J. Tobias, and M. L. Klein, Mol. Phys. 87, 1117 (1996).

${ }^{47}$ Y. Liu and M. E. Tuckerman, J. Chem. Phys. 112, 1685 (2000).

${ }^{48}$ I. Vattulainen, S. C. Ying, T. Ala-Nissila, and J. Merikoski, Phys. Rev. B 59, 7697 (1999).

${ }^{49}$ T. Ala-Nissila, R. Ferrando, and S. C. Ying, Adv. Phys. 51, 949 (2002).

${ }^{50} \mathrm{P}$. J. Flory, Statistical Mechanics of Chain Molecules (Wiley Interscience, New York, 1969), pp. 16-17.

${ }^{51}$ G. W. N. White, S. Goldman, and C. G. Gray, Mol. Phys. 98, 1871 (2000).

${ }^{52}$ E. Beerdsen, D. Dubbeldam, and B. Smit (unpublished).

${ }^{53}$ R. M. Barrer and H. Villiger, Z. Kristallogr. 128, 352 (1969).

${ }^{54}$ J. J. Pluth and J. V. Smith, J. Am. Chem. Soc. 102, 4707 (1980).

${ }^{55}$ H. L. Tepper and W. J. Briels, J. Chem. Phys. 116, 9464 (2002).

${ }^{56}$ A. F. Voter and J. D. Doll, J. Chem. Phys. 82, 80 (1985).

${ }^{57}$ D. Dubbeldam, E. Beerdsen, S. Calero, and B. Smit (unpublished).

${ }^{58}$ E. Beerdsen, B. Smit, and S. Calero, J. Phys. Chem. B 106, 10659 (2002).

${ }^{59}$ S. Calero, D. Dubbeldam, R. Krishna, B. Smit, T. J. H. Vlugt, J. F. M. Denayer, J. A. Martens, and T. L. M. Maesen, J. Am. Chem. Soc. 126, 11377 (2004).

${ }^{60}$ P. Demontis, G. B. Suffritti, S. Quartieri, E. S. Fois, and A. Gamba, Zeolites 7, 522 (1987).

${ }^{61}$ P. Demontis, G. B. Suffritti, S. Quartieri, E. S. Fois, and A. Gamba, J. Phys. Chem. 92, 867871 (1988). 